


\section{Effects of a high level of dietary zinc as model intervention on intestinal microbiota and mucosal gene expression in piglets}

A.J.M. Jansman, D. Schokker, R. Gerritsen, F. de Bree, M.M. Hulst, M.A. Smits

This research was conducted by Wageningen Livestock Research, within the framework of the public private partnership "Feed4Foodure" and partially funded by the Ministry of Economic Affairs (Policy Support Research; project number BO -22.04-002-001)

Wageningen Livestock Research

Wageningen, December 2016

Report 1000 


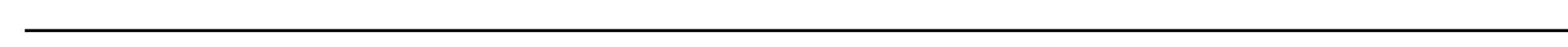


Jansman, A.J.M., D. Schokker, R. Gerritsen, F. de Bree, M.M. Hulst, M.A. Smits, 2016. Effects of a high level of dietary zinc as model intervention on intestinal microbiota and mucosal gene expression in piglets.

Wageningen Livestock Research, Report 1000.

This report can be downloaded for free at http://dx.doi.org/10.18174/401707 or at www. wur. nl/livestock-research (under Wageningen Livestock Research publications).

(C) 2016 Wageningen Livestock Research

P.O. Box 338, 6700 AH Wageningen, The Netherlands, T +31 (0)317 483953 ,

E info.livestockresearch@wur.nl, www.wur.nl/livestock-research. Wageningen Livestock Research is part of Wageningen University \& Research.

All rights reserved. No part of this publication may be reproduced and/or made public, whether by print, photocopy, microfilm or any other means, without the prior permission of the publisher or author.

$\rho$ The ISO 9001 certification by DNV underscores our quality level. All our research commissions are in line with the Terms and Conditions of the Animal Sciences Group. These are filed with the District Court of Zwolle.

Wageningen Livestock Research Report 1000 


\section{Table of contents}

$\begin{array}{ll}\text { Summary } & 5\end{array}$

1

$\begin{array}{ll}\text { Introduction } & \mathbf{7}\end{array}$

2

$\begin{array}{ll}\text { Material \& methods } & 8\end{array}$

2.1 Experimental treatments $\quad 8$

2.2 Piglets, housing and feeding $\quad 8$

$\begin{array}{lll}2.3 & \text { Observations, measurements and sampling } & 10\end{array}$

2.4 Microbiota and gene expression analysis 11

2.4.1 Microbiota analysis 11

2.4.2 Transcriptomics analysis 11

2.5 Statistical analysis $\quad 12$

3

$\begin{array}{ll}\text { Results } & 13\end{array}$

3.1 Course of the study and zootechnical performance $\quad 13$

3.2 Concentrations Zn, haptoglobin and cytokines in plasma 14

3.3 Microbiota composition in digesta 16

$\begin{array}{lll}3.4 & \text { Gene expression in intestinal tissue } & 21\end{array}$

$\begin{array}{lrr}4 & \text { Discussion } & 27\end{array}$

$\begin{array}{llr}5 & 31\end{array}$

$\begin{array}{llr}6 & \text { Appendices } & 33\end{array}$ 


\section{Summary}

Development of immune competence in pigs is important for resilience, disease resistance, and performance later in life. Dietary interventions in early life can contribute to immune system development. Use of model dietary interventions, such as diets with a high concentration of zinc, can contribute to the understanding of the interactions between the diet, the intestinal microbiota and intestinal tissues. The aim of the present study was to evaluate the effects of provision of a diet with a high concentration of zinc ( $\mathrm{Zn}, 2690 \mathrm{mg} / \mathrm{kg}$ ), as model intervention, as compared to provision of a control diet with a regular $\mathrm{Zn}$ concentration $(100 \mathrm{mg} / \mathrm{kg}$ ) during a period of nine days during the post weaning period ( $d$ 14-23 post weaning $(\mathrm{pw})$ ), on the composition of the intestinal microbiota and gene expression in the intestinal mucosa on d 23 and 35 pw (12 d after the termination of the $\mathrm{Zn}$ intervention).

Feed intake and body weight gain were higher in the high zinc group between $\mathrm{d} 14$ and 23 compared to the control group, but lower in the period after the dietary intervention (d 23-35). The feed conversion ratio (FCR) over the complete experimental period ( $d$ 0-35 pw) did not differ between treatment groups. The high zinc treatment increased plasma zinc concentration of piglets on $\mathrm{d} 23$, but values returned to the level of the control group on d $35 \mathrm{pw}$. There was no treatment or time effect on the concentrations of the acute phase protein haptoglobin and the cytokines TNF- $\alpha$ and IL- 6 on d 23 and 35 pw, except for a higher concentration of TNF-a on d 23 pw in the high Zn group $(P<0.05)$.

On d 14, 23 and 35 significant differences in the microbiota composition were observed between jejunal and ileal digesta. In jejunal digesta, Lactobacillaceae was the most dominant family, followed by Turibacteraceae and Streptococcaceae. In ileal digesta Lactobacillaceae and Turibacteraceae were the most dominant microbial families. On d 23 pw in jejunal digesta, some clustering occurred related to microbiota composition of samples between treatment groups. On d 35, no clustering of samples of treatment groups was observed anymore. On d 23 pw in ileal digests also clustering occurred of the samples between treatment groups, in particular of the high zinc group. On d 35, clustering of samples belonging to the low zinc treatment was still observed. Based on the Chao- 1 index, a significant effect of time (age of the piglets) was observed on the ileal microbiota diversity $(P<0.001)$ and a significant age $x$ dietary treatment interaction $(P<0.01)$. The diversity was highest on $d 35$ and lowest on $\mathrm{d} 14$, while on $\mathrm{d} 23$ the diversity tended to be higher in piglets receiving the high zinc diet from d 14-23, compared to the diversity on d 23 of the animals receiving the control diet $(P<0.10)$.

Whole genome gene expression analysis of the jejunal and ileal mucosa revealed a small overall timetreatment interaction effect at d 23 pw in both intestinal tissues. In both jejunal and ileal mucosa various genes/probes were differentially up or down regulated between treatments. In intestinal tissue samples obtained on d 35 no differences in gene expression were observed. Probes of d 23 pw that were differently expressed and had an annotation were subsequently used as input for further functional analysis using DAVID and Gene Decks databases. The analysis did not result in the identification of gene sets that were differentially expressed between dietary treatments. However, it was shown that a number of upregulated genes in the jejunal mucosa on d 23 pw by the high zinc intervention are involved in pathways related to mineral absorption, immune signalling and cell energy metabolism (glycolysis and gluconeogenesis). A few down regulated genes by the $\mathrm{Zn}$ intervention are also involved in immune signalling pathways.

It was concluded from the present study that provision of a diet with a high concentration of zinc as zinc oxide during a short period of time ( 9 days) to piglets in the post weaning period ( $d$ 14-23 pw) induces differences intestinal microbiota composition, in particular in the ileum, and on the intestinal expression of genes in part related to the functioning of the local innate immune system. The high dietary zinc intervention can therefore be considered as a suitable model for studying relationships between dietary interventions, intestinal microbiota composition and development of immune competence in post weaning piglets. 


\section{Introduction}

The health status of farm animals is in part determined by the competence and responses of the immune system. Both determine the resilience of animals towards environmental stressors and to challenge by pathogenic micro-organisms. Responses of the immune system need to be expressed at the proper location in the animal and balanced in time. Modulation of the development of the immune system and its competence to express proper, well balanced responses later in life via dietary interventions is an increasing area of research in both the human and animal domain. Via the development and application of innovative dietary interventions and feeding strategies support can be given to the development of the gastrointestinal tract in pigs and poultry with regard to both its absorption and barrier function, including the competence of the (local) immune system. A proper functioning of the immune system largely contributes to the support of animal health and their productive performance under a range of environmental conditions.

The research programme Nutrition, Gut Health and Immunity (VDI), as part of the public private partnership Feed4Foodure (F4F), is focussed on the development and validation of in vitro, ex vivo and in vivo models to study the effects of dietary interventions in pigs, poultry and veal calves on the development of immune competence. One of the research lines within the programme has the aim to develop models for studying the effects of dietary interventions in piglets from two weeks after weaning onwards on the development of immune competence and to identify indicators for function, health and mucosal immunity of/in the gut. These indicators are useful for the evaluation of dietary interventions on gut health and function in future research.

High dietary concentrations of zinc were shown to change the composition, diversity and activity of the intestinal microbiota in piglets in the immediate post-weaning period (Vahjen et al., 2010, 2011; Hojberg et al., 2005) and can contribute to maintaining gut and animal health in the critical post weaning phase. It was also shown that a high concentration of $\mathrm{ZnO}$ in the diet influences the activity of intestinal brush border bound alkaline phosphatase in a dose-dependent manner, however, did not affect the activity of other brush border enzymes involved in nutrient digestion (Martin et al., 2013). In addition, Sargeant et al. (2010) showed that a high concentration of dietary zinc reduced the expression of immune related genes involved in inflammatory processes in gut tissue after challenge with a pathogenic E. coli (ETEC). In another study, a similar high zinc intervention via the diet induced changes in the intestinal expression of proteins involved in oxidative stress, cell differentiation and apoptosis (programmed cell death) (Wang et al., 2009).

The former suggests that the dietary zinc concentration of piglet diets interferes with the development of both the intestinal microbiota and the (local) immune system. It was concluded that provision of a high zinc diet for a short period of time after the immediate post-weaning phase of piglets was a suitable "model intervention" to study the possibilities to influence immune competence of pigs via the modification of intestinal microbiota and functional development of intestinal tissue in the framework of the VDI research programme.

The aim of the present study was to evaluate the effects of provision of a diet with a high concentration of zinc $(\mathrm{Zn}, 2690 \mathrm{mg} / \mathrm{kg}$ ), compared to a control diet with a regular $\mathrm{Zn}$ concentration $(100 \mathrm{mg} / \mathrm{kg}$ ), as a model intervention during a period of nine days during the post weaning period ( $\mathrm{d}$ 14-23 post weaning), on the composition of the intestinal microbiota and gene expression in the intestinal mucosa on d 23 and 35 ( 12 days after the termination of the dietary $\mathrm{Zn}$ intervention). 


\section{Material \& methods}

\section{$2.1 \quad$ Experimental treatments}

In the study two experimental treatments were evaluated.

I. Control treatment. Piglets were fed with a control diet with $100 \mathrm{mg} / \mathrm{kg}$ Zn over the period from d 14-35 post weaning.

II. High zinc treatment. Piglets were fed with a experimental diet with $2690 \mathrm{mg} \mathrm{Zn/kg} \mathrm{diet} \mathrm{from} \mathrm{d}$ 14-23 and a control diet with $100 \mathrm{mg} \mathrm{Zn}$ per $\mathrm{kg}$ from d 23-35 post weaning.

The piglets in both treatment groups received a weaning diet from d 0-14 with $111 \mathrm{mg} \mathrm{Zn} \mathrm{per} \mathrm{kg.}$

The study was performed with three pens with 12 piglets per pen in both treatments, in total comprising 72 piglets.

\subsection{Piglets, housing and feeding}

The study was performed in the experimental facilities of Agrifirm "Laverdonk" in Heeswijk Dinther, The Netherlands.

The piglets were of the Tempo $x$ Topigs 20 genotype. At the start of the study the piglets were divided per pen in a way that the mean body weight and its variation per pen were similar.

The piglets were weaned at a mean age of $28 \pm 1.5 \mathrm{~d}$. The piglets received creep feed during the suckling period. The mean body weight of the piglets one day prior to the start of the study was 7.9 $\mathrm{kg}$.

The piglets were housed in floor pens $(1.75 \times 3.00 \mathrm{~m})$ with 12 piglets per pen. Male (boars) and female piglets were equally distributed over pens and litter mates were divided equally over pens as far as possible. The piglets were fed ad libitum using a dry feed dispenser. The diets were provided as pellets. The animals had free access to water via an automatic drinking device.

The ingredient and calculated nutrient composition of the weaning diet and the starter diet (control) are given in Tables 1 and 2 . The diets were formulated to be nutritionally adequate using data on the composition and nutritional value of feed ingredients according to CVB (2011).

After weaning, the piglets were fed the same weaning diet during the period of $d 0$ till 14 . From $d 14$ till 23 after weaning the experimental piglet diets were provided containing either a regular or a high zinc concentration (analysed zinc concentrations 100 and $2690 \mathrm{mg} / \mathrm{kg}$, respectively). The contrast in $\mathrm{Zn}$ concentration was realised by supplementing $\mathrm{ZnO}$ to the control diet. From d 23 till 35 the piglets in both treatment groups received the same starter piglet diet with a regular $\mathrm{Zn}$ concentration (analysed $100 \mathrm{mg} \mathrm{Zn}$ per $\mathrm{kg}$ ). 
Table 1. Calculated ingredient and nutrient composition of the weaning diet and the control experimental starter diets $(\mathrm{g} / \mathrm{kg})$.

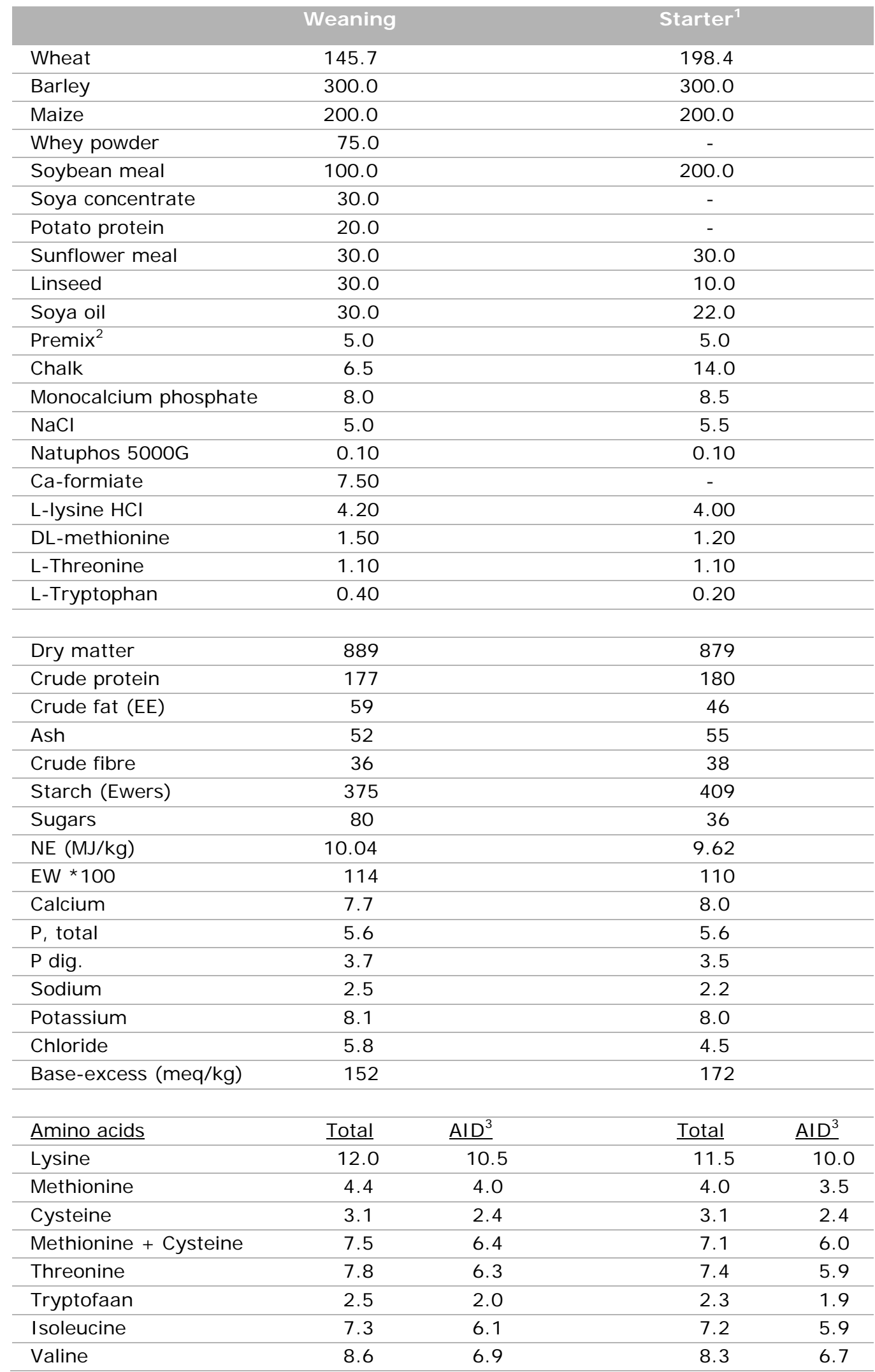

${ }^{1}$ The high zinc starter diet was formulated by additionally supplementing $\mathrm{Zn}$ as $\mathrm{ZnO}$ to obtain a diet with $2690 \mathrm{mg} \mathrm{Zn}$ per $\mathrm{kg}$. ${ }^{2}$ Both the weaning and the starter diets contained $20 \mathrm{mg} / \mathrm{kg}$ supplemented $\mathrm{Cu}$.

${ }^{3} \mathrm{AID}$ : apparent ileal digestible 
Table 2. Analysed nutrient composition of the experimental starter diets (d 14-35).

\begin{tabular}{lcc} 
& Control & High Zn \\
\hline Zn $(\mathrm{mg} / \mathrm{kg})$ & 100 & 2690 \\
\hline Moisture & 112 & 112 \\
\hline Ash & 55 & 54 \\
\hline Crude protein $(\mathrm{N} \times 6.25)$ & 181 & 182 \\
\hline Crude fibre & 37 & 36 \\
\hline Sugars & 40 & 39 \\
\hline Crude fat (EE) & 49 & 48 \\
\hline Starch (Ewers) & 419 & 417 \\
\hline
\end{tabular}

\subsection{Observations, measurements and sampling}

- The weaning and the experimental diets were analysed on the concentrations of dry matter, ash, $\mathrm{N}$ (crude protein), crude fat (EE), crude fibre, starch [Ewers] and sugars using standard methods.

- The piglets were weighed on $d-1,14,23$ en 35 post weaning

- The feed intake per pen was registered over the periods of d 0-14, 14-23 and 23-35.

- The mean faeces consistency score per pen was judged on a scale of 1 till 3 three times per week during the experimental period ( $\mathrm{d} 0-35)$.

- On d 14, 23 and 35 per pen one (d 14) or two piglets per pen ( $d 23$ and 35) were euthanized to obtain samples of intestinal digesta (duodenum, jejunum, ileum, en colon) for (optional) microbiota composition, intestinal mucosa (jejunum and ileum) for gene expression measurements, intestinal tissue (jejunum and ileum) for optional immuno-histochemical analyses, and blood.

- Plasma samples obtained at dissection of piglets on d 14, 23 and 35 were analysed on concentrations haptoglobin, IL- 6 en TNF- $a$ using commercial ELISA kits according to instructions of the manufacturer (MT-diagnostics Etten-Leur, The Netherlands). 


\subsection{Microbiota and gene expression analysis}

\subsubsection{Microbiota analysis}

\section{DNA Extraction}

Samples of jejunal and ileal digesta were snap frozen in liquid nitrogen and stored at $-80^{\circ} \mathrm{C}$. For the microbial DNA extraction the following protocol was handled:
1) Digesta was mixed 1:1 with PBS
2) 10 glass beads were added
3) Vortex
4) Spin for $5 \min 300 \mathrm{~g} 4{ }^{\circ} \mathrm{C}$
5) Supernatant to new tube
6) Spin for $10 \mathrm{~min} 9000 \mathrm{~g} 4{ }^{\circ} \mathrm{C}$
7) Remove supernatant
8) Follow QI Aamp DNA Stool Mini Kit protocol as described
9) Elute in $100 \mu \mathrm{l}$ of (provided) elute buffer
10) Optical Density measurement on Nanodrop (Agilent Technologies) to check quality

\section{V3 PCR}

Oligo V3_F, with sequence ; CCTACGGGAGGCAGCAG and oligo V3_R with sequence; ATTACCGCGGCTGCTGG

The following PCR program was run:

1) $98^{\circ} \mathrm{C} 2 \mathrm{~min}$

2) $98^{\circ} \mathrm{C} 10 \mathrm{~s}$

3) $55^{\circ} \mathrm{C} 30 \mathrm{~s}$

4) $72{ }^{\circ} \mathrm{C} 10 \mathrm{~s}$

5) $72{ }^{\circ} \mathrm{C} 7 \mathrm{~min}$

Where step 2-4 were repeated for 15 times. All PCR products were subjected to quality control by running $5 \mu \mathrm{l}$ of CPR product on a $2 \% \mathrm{E}$-Gel ${ }^{\circledR}$ Agarose Gel Electrophoresis System (Life Technologies).

\section{QIIME}

Samples were sequenced by targeted-amplicon $16 \mathrm{~S}-\mathrm{V} 3$ sequencing and taxonomy summaries per sample were analysed using QIIME [12]. Standard assembly based on amplicon, with primer removal, was performed. For Quality filtration the following settings were used: 1) >Q20 and 2) amplicons $>100$ bases. For the data analysis pseudoreads were clustered into operational taxonomic units (OTUs) per sample at $97 \%$ similarity and OTU-representative sequences were aligned against the aligned Greengenes core set (13_8 release) $[13,14]$. Furthermore chimeras were removed with Chimeraslayer [15].

\section{Statistical analysis}

The biodiversity was calculated by the vegan package (http://cran.r-project.org/web/ packages/vegan/) within the R environment, by employing the Shannon and Chao- 1 diversity indices. The Redundancy analysis (RDA) was also performed by using the vegan package, the following model was ran on the family level microbiota data: $Y=$ Time (day) + Treatment + Time (day) * Treatment + error. Furthermore, statistical significance testing for over- and under-representation of the bacterial groups was made at the family level by performing the Wilcoxon signed-rank test, and P-values were converted to false discovery rate (FDR) values to correct for multiple testing.

\subsubsection{Transcriptomics analysis}

RNA Extraction Tissue

Total RNA was extracted from 50 to $100 \mathrm{mg}$ tissue mucosal scrapings of jejunum and ileum. The jejunum and ileum samples were homogenised using the TisuPrep Homogenizer Omni TP TH220P) in TRizol reagent (Life Technologies) as recommended by the manufacturer with minor modifications. The homogenised tissue samples were dissolved in $5 \mathrm{ml}$ of TRizol reagent. After centrifugation the 
supernatant was transferred to a fresh tube. Subsequently a phase separation with chloroform was performed as described by the manufacturer Life Technologies. The RNA was precipitated and dissolved and quantified by absorbance measurements at $260 \mathrm{~nm}$. Quality Control was performed by Agilent Bioanalyser.

Labelling, Hybridization, Scanning and Feature Extraction

Labelling of RNA was done as recommended by Agilent Technologies using the One-Color MicroarrayBased Gene Expression Analysis Low input Quick Amp Labelling. The input was 10 ng of total RNA and $600 \mathrm{ng}$ of labelled cRNA was used on the eight pack array.

Hybridization was performed as described in the One-Color Microarray-Based Gene Expression Analysis Low input Quick Amp Labelling protocol from Agilent in the hybridization oven (G2545A hybridization Oven Agilent Technologies). The hybridization temperature was $65^{\circ} \mathrm{C}$ with rotation speed 10 rpm for 17 hours. After 17 hours the arrays were washed as described in the One-Color MicroarrayBased Gene Expression Analysis Low input Quick Amp Labelling protocol from Agilent.

The arrays were scanned using the DNA microarray scanner with Surescan high resolution Technology from Agilent Technologies. Agilent Scan Control with resolution of $5 \mu \mathrm{m}, 16$ bits and PMT of $100 \%$. Feature extraction was performed using protocol 10.7.3.1 (v10.7) for one colour gene expression.

\section{Data analysis}

The data were analysed by using R (v3.0.2) by executing different packages, including LIMMA and arrayQualityMetrics. The data were read in and background corrected (method="normexp" and offset $=1$ ) with functions from the R package LIMMA [1] from Bioconductor [2]. Quantile normalisation of the data was performed between arrays. The duplicate probes mapping to the same gene were averaged ('avereps') and subsequently the lower percentile of probes was removed in a three-step procedure, 1) get the highest of the dark spots to get a base value, 2) multiply by 1.1 and 3) the gene/probe must be expressed in each of the samples in an experimental condition (e.g. in ileum d 14 control).

\subsection{Statistical analysis}

The results for body weight gain, daily feed intake, feed conversion ratio and plasma zinc concentrations were statistically analysed by ANOVA (Genstat 5, release 3; Payne et al., 1993). The data were analysed using "dietary treatment" as main experimental factor in the statistical model.

An effect of a factor in the statistical evaluation was considered to be significant when the probability of having no effect was less than $5 \%(P<0.05)$. Differences between treatment means were evaluated further using the Least Significant Difference test (Snedecor and Cochran, 1980).

Details regarding the statistical evaluation of data on the microbiota composition in digesta and gene expression in intestinal mucosa tissues are provided above and in the chapter Results. 


\section{Results}

\subsection{Course of the study and zootechnical performance}

The study generally went well. No specific health problems were encountered during the study.

Data on the development in body weight and the zootechnical performance of the piglets per experimental group throughout the study are provided in Table 3. The mean body weight of the piglets at the start of the study of both treatment groups was identical while the body weight on $d 35$ pw was $0.6 \mathrm{~kg}$ higher in the control group compared to the intervention group.

Feed intake and body weight gain were higher in the high zinc group between d 14 and 23 pw compared to the control group, but lower in the period after the dietary intervention ( $d$ 23-35 pw). The feed conversion ratio (FCR) over the complete experimental period ( $\mathrm{d}$ 0-35 pw) did not differ between treatment groups, but FCR was slightly in favour of animals in the high zinc group during the intervention period ( $\mathrm{d}$ 14-23 pw).

Table 3. Body weight (BW) on $d-1,14,23$ and 35, body weight gain (BWG), feed intake and feed conversion ratio of the pigs over the periods $d 0-14,14-23,23-35$ and $d 0-35$ of the study.

Control High zinc P

\begin{tabular}{lccc}
\hline BW (kg) & & & \\
\hline d -1 & 7,9 & 7,9 & 1.00 \\
\hline d 14 & 10,9 & 10,9 & 0.99 \\
\hline d 23 & 14,5 & 14,8 & 0.83 \\
\hline d 35 & 20,7 & 20,1 & 0.75 \\
\hline BWG (g/ d) & & & \\
\hline d 0-14 & 213 & 216 & 0.90 \\
\hline d 14-23 & 400 & 440 & 0.05 \\
\hline d 23-35 & 524 & 475 & 0.27 \\
\hline d 0-35 & 368 & 362 & 0.83 \\
\hline Feed intake (g/ d) & & & 0.84 \\
\hline d 0-14 & 261 & 267 & 0.38 \\
\hline d 14-23 & 575 & 609 & 0.35 \\
\hline d 23-35 & 771 & 709 & 0.79 \\
\hline d 0-35 & 517 & 506 & \\
\hline FCR & & & 0.81 \\
\hline d 0-14 & 1.226 & 1.232 & 0.52 \\
\hline d 14-23 & 1.436 & 1.390 & 0.60 \\
\hline d 23-35 & 1.471 & 1.493 & 0.77 \\
\hline d 0-35 & 1.404 & 1.397 & \\
\hline
\end{tabular}

Overall the faecal consistency score in both treatment groups was close to 1 suggesting no deviations from normal and no differences between treatment groups (Table 4). 
Table 4. Mean faecal consistency score ${ }^{1}$ of the pigs per treatment group during the course of the study.

\begin{tabular}{lcc} 
d $0-14$ & Control & High zinc \\
\hline d $14-23$ & 1.12 & 1.13 \\
\hline d 23-35 & 1.09 & 1.09 \\
\hline
\end{tabular}

${ }^{1}$ scored per pen on a scale of 1 (normal faecal consistency) to 3 (diarrhoea).

\subsection{Concentrations $\mathrm{Zn}$, haptoglobin and cytokines in plasma}

The plasma concentration of $\mathrm{Zn}$ was measured in the piglets on $\mathrm{d} \mathrm{14,23}$ and 35. Results are presented in Figure 1. On d 14 prior to the experimental treatment, there was no difference in blood Zn concentration between treatment groups. On d 23, the zinc concentration in plasma was significantly higher in the piglets receiving the high zinc diet $(P<0.05)$. On $d 35$, twelve days after having fed the same low zinc starter diet to both experimental groups, piglets in both treatment groups had on average a similar blood $\mathrm{Zn}$ concentration again.

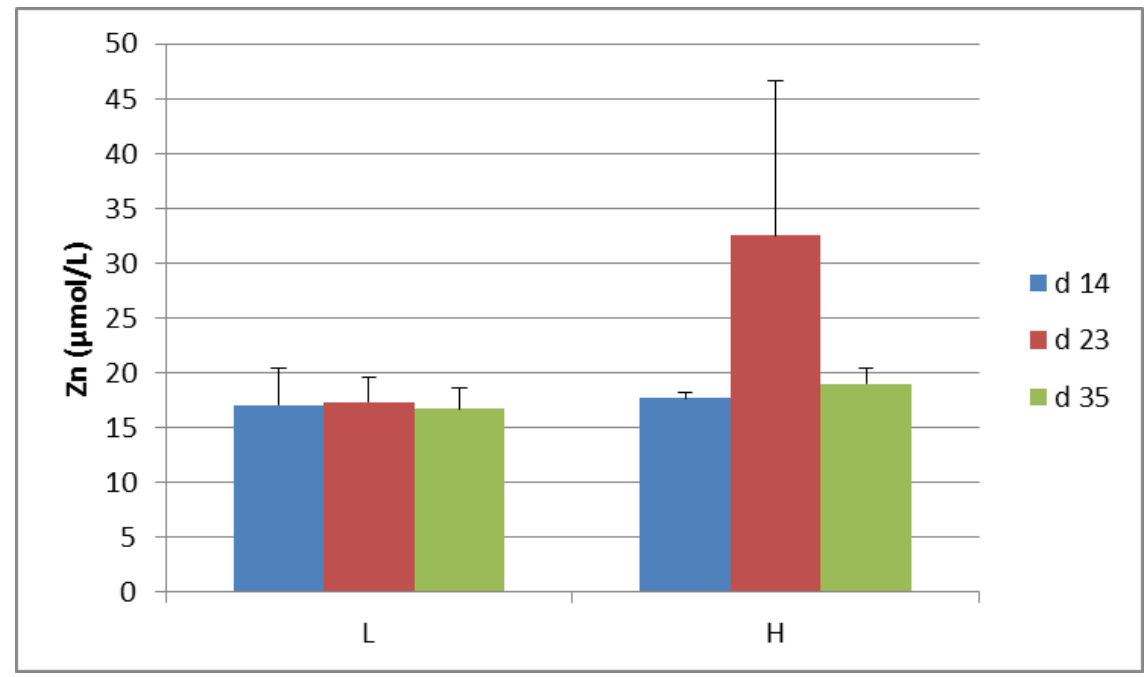

Figure 1. Effect of the experimental treatments on the concentration of Zn in plasma on d 14, 23 and 35.

The concentrations haptoglobin, TNF- $\mathrm{a}$ and IL-6 were measured in blood plasma on d 14, 23 and 35. The results are presented in Figure 2 and Table 5. There were no significant differences between the experimental treatments in the concentration of the acute phase protein haptoglobin and the cytokines, except for a higher concentrations of TNF- $\alpha$ on d 23 in the high Zn group $(P<0.05)$. In the control group there was no effect of time (day) on the plasma concentration of either of the three immune proteins. Their concentrations were generally relatively low and substantial between animal variation in plasma concentrations was observed. 

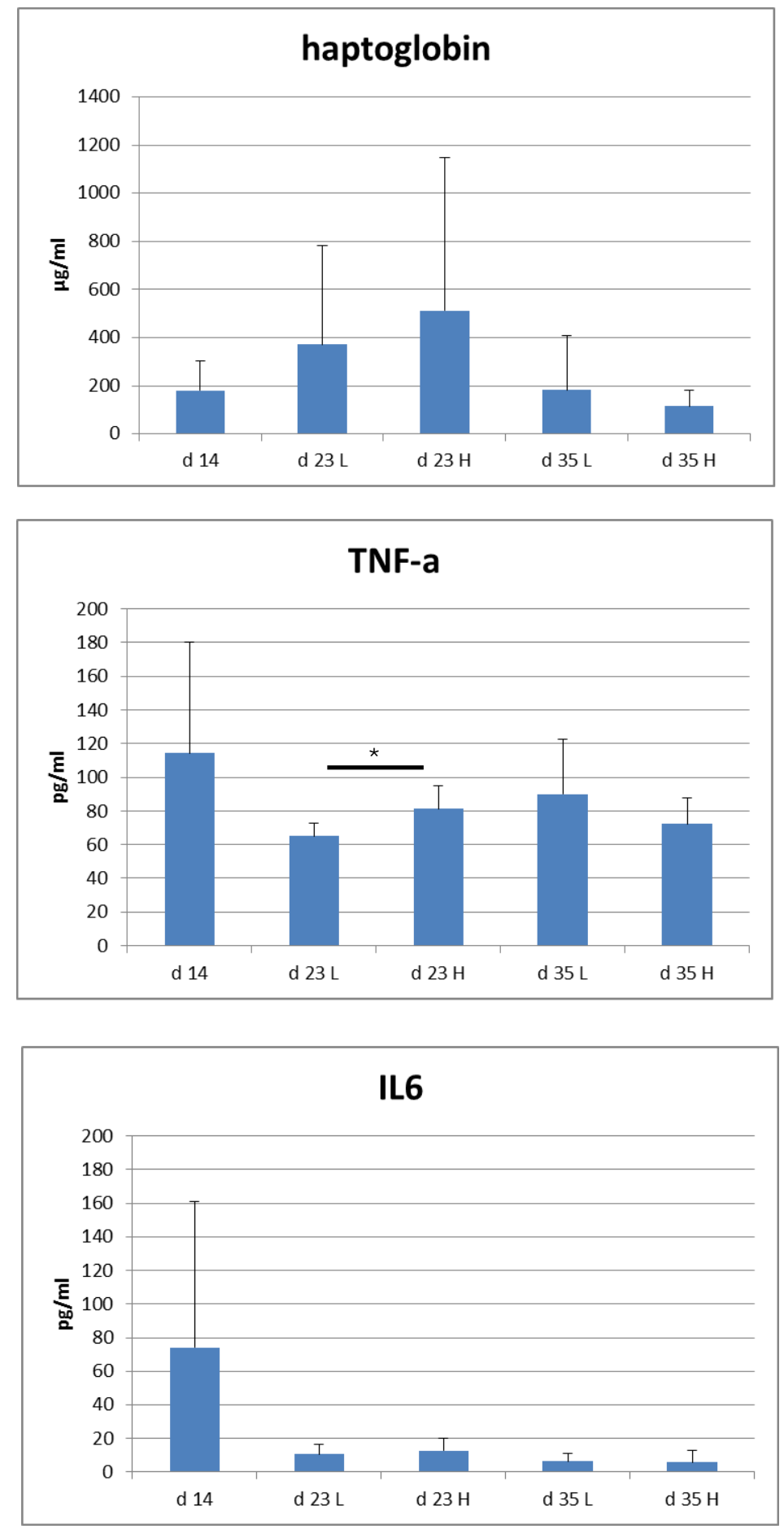

Figure 2. Effects of the experimental treatments on the concentration of haptoglobin, TNF- $a$ and IL- 6 in plasma on d 14, 23 and 35 (means \pm sd values). 
Table 5. Concentrations of IL-6, TNF- $a$ and haptoglobin in plasma on d 14, 23 and 35.

\begin{tabular}{|c|c|c|c|c|}
\hline & Control & High zinc & LSD & P-value \\
\hline \multicolumn{5}{|l|}{ I L-6 (pg/ml) } \\
\hline d 14 & $2.1^{1}$ & & & \\
\hline d 23 & 5.4 & 8.5 & 9.0 & 0.44 \\
\hline d 35 & 4.3 & 4.0 & 7.1 & 0.93 \\
\hline Day $x$ Treatment & & & 8.1 & 0.54 \\
\hline \multicolumn{5}{|l|}{ TNF- $\alpha(p g / m l)$} \\
\hline d 14 & 114 & & & \\
\hline d 23 & $65^{a}$ & $81^{b}$ & 14 & 0.03 \\
\hline d 35 & 90 & 72 & 34 & 0.27 \\
\hline Day $x$ Treatment & & & 23 & 0.05 \\
\hline \multicolumn{5}{|c|}{ Haptoglobin $(\mu \mathrm{g} / \mathrm{ml})$} \\
\hline d 14 & 180 & & & \\
\hline d 23 & 371 & 512 & 762 & 0.68 \\
\hline d 35 & 182 & 115 & 213 & 0.48 \\
\hline Day $x$ Treatment & & & 483 & 0.53 \\
\hline
\end{tabular}

\subsection{Microbiota composition in digesta}

On d 14, 23 and 35 the microbiota composition in jejunal and ileal digesta of piglets of both treatment groups was determined. In Figure 3 results of a clustering analysis are presented over all digesta samples analysed. The analysis showed that no clear discrimination could be made at genus level between jejunal and ileal digesta samples. Dominant taxa at genus level over time points in jenunal digesta are Lactobacillus, Streptococcus, and Turicibacter and in ileal digesta are Lactobacillus, Streptococcus and Staphylococcus.
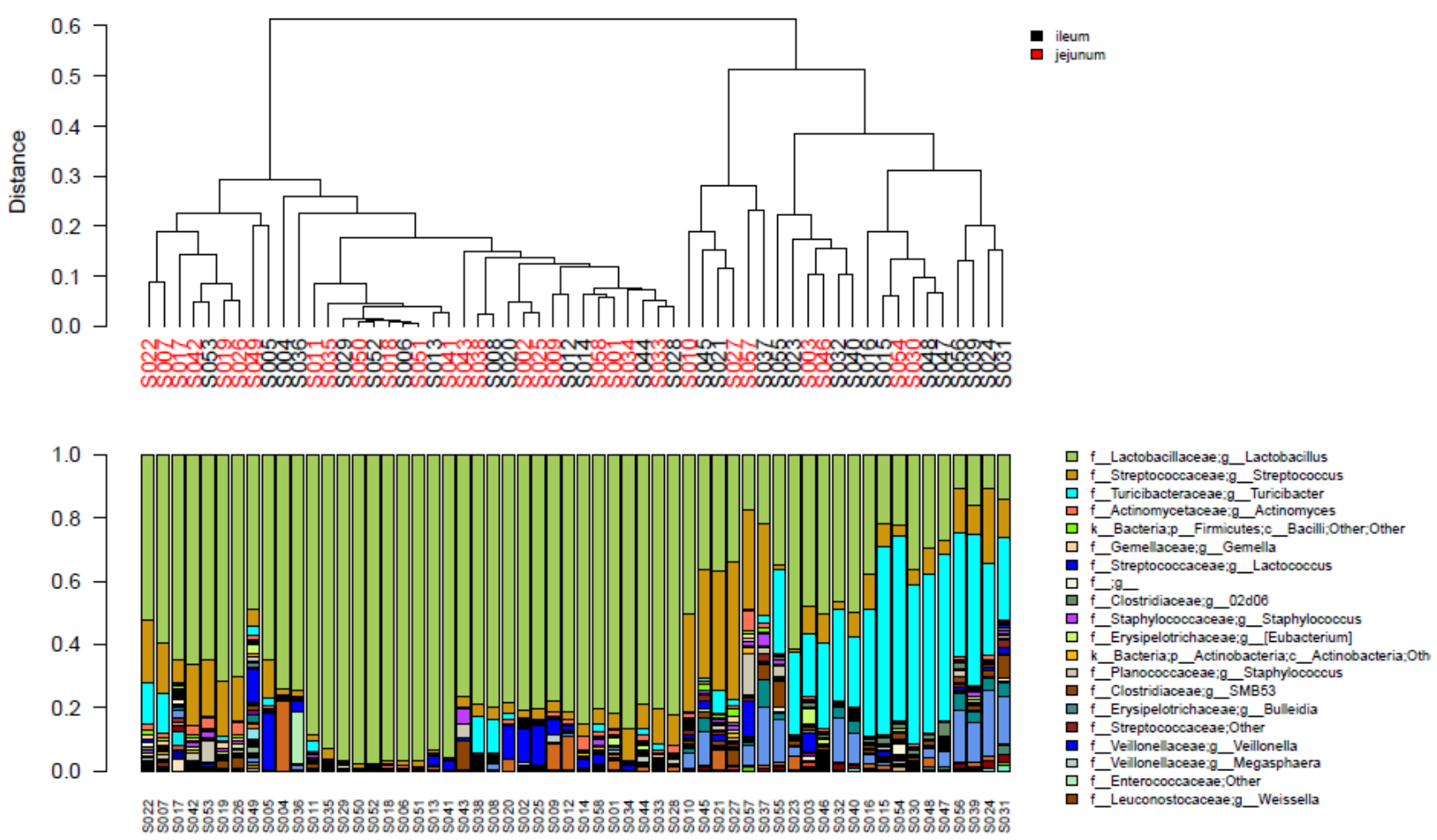

Figure 3. Microbiota composition at genus level in jejunal and ileal digesta over the experimental treatments and time points of measurement ( $d 14,23$ and 35). 
A further clustering analysis over sampling time points and experimental treatments (high zinc vs. normal zinc) was made. Results are presented in Figures 4 and 5 for the microbiota composition data in jejunal and ileal digesta, respectively.

In jejunal digesta, Lactobacillaceae was the most dominant family in most samples, followed by Turibacteraceae and Streptococcaceae. Some clustering occurred of the samples between treatment groups on d 23, after nine days of feeding of either a normal or a high zinc diet. On d 35, no clustering of treatment groups was observed anymore.

In ileal digesta samples Lactobacillaceae and Turibacteraceae were the most dominant microbial families. Also here, clustering occurred of the samples between treatment groups on d 23 in particular of the high zinc group, after nine days of feeding either a normal or a high zinc diet. On d 35, clustering of samples belonging to the low zinc treatment was still observed although both groups were fed the same diet for the previous twelve days.

Further data on the microbiota composition (relative contribution at genus level) per time point (age) and dietary treatment in jejunal and ileal digesta are provided in Appendices 1 and 2. 


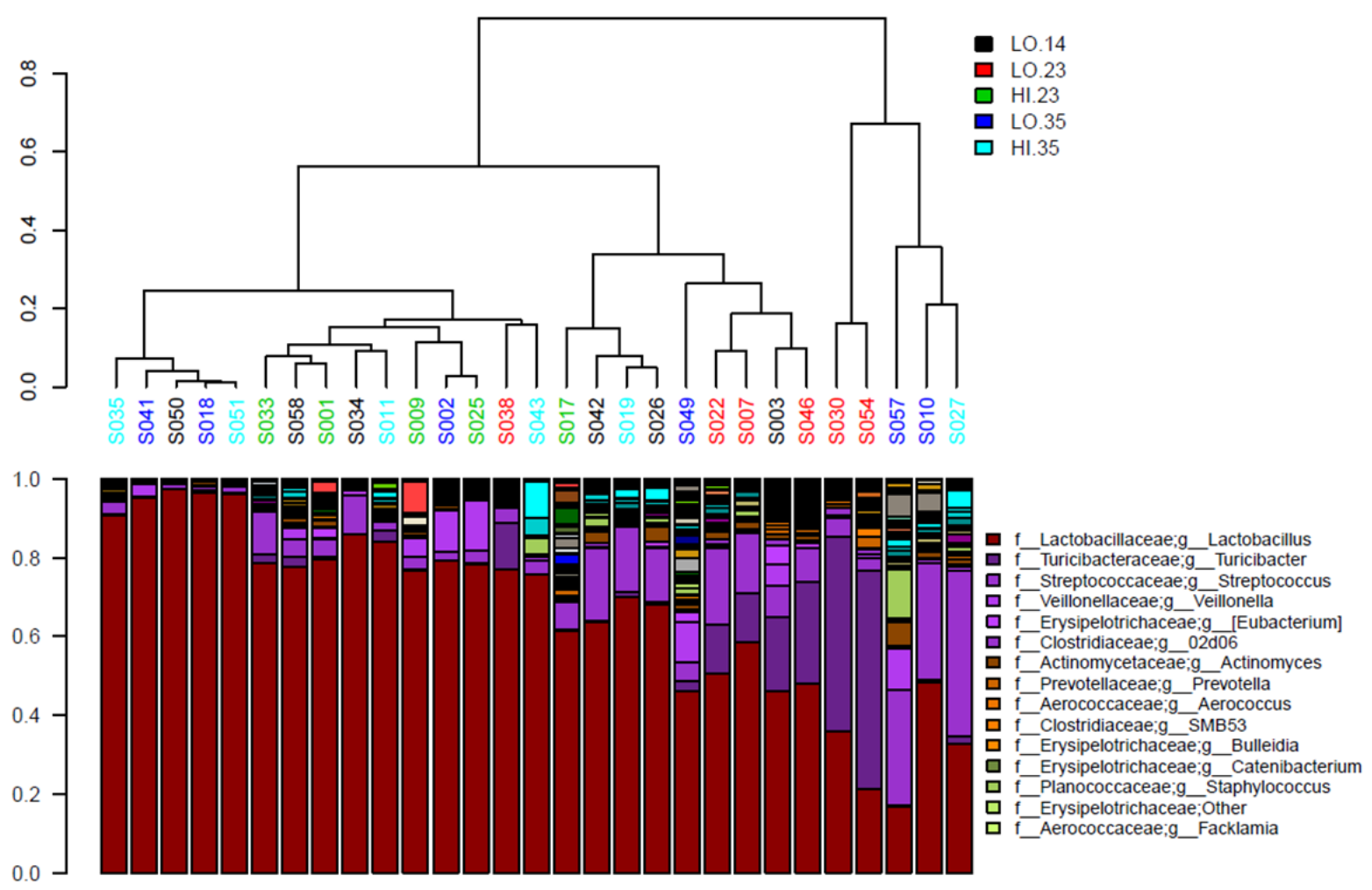

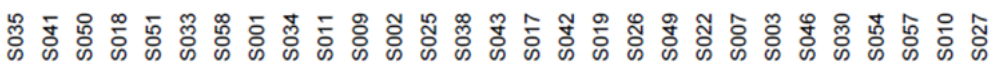

Figure 4. Cluster analysis of microbiota composition at genus level as analysed in jejunal digesta and affected by treatment (control - LO; high zinc - HI) and time point of measurement (d 14, 23 and 35).
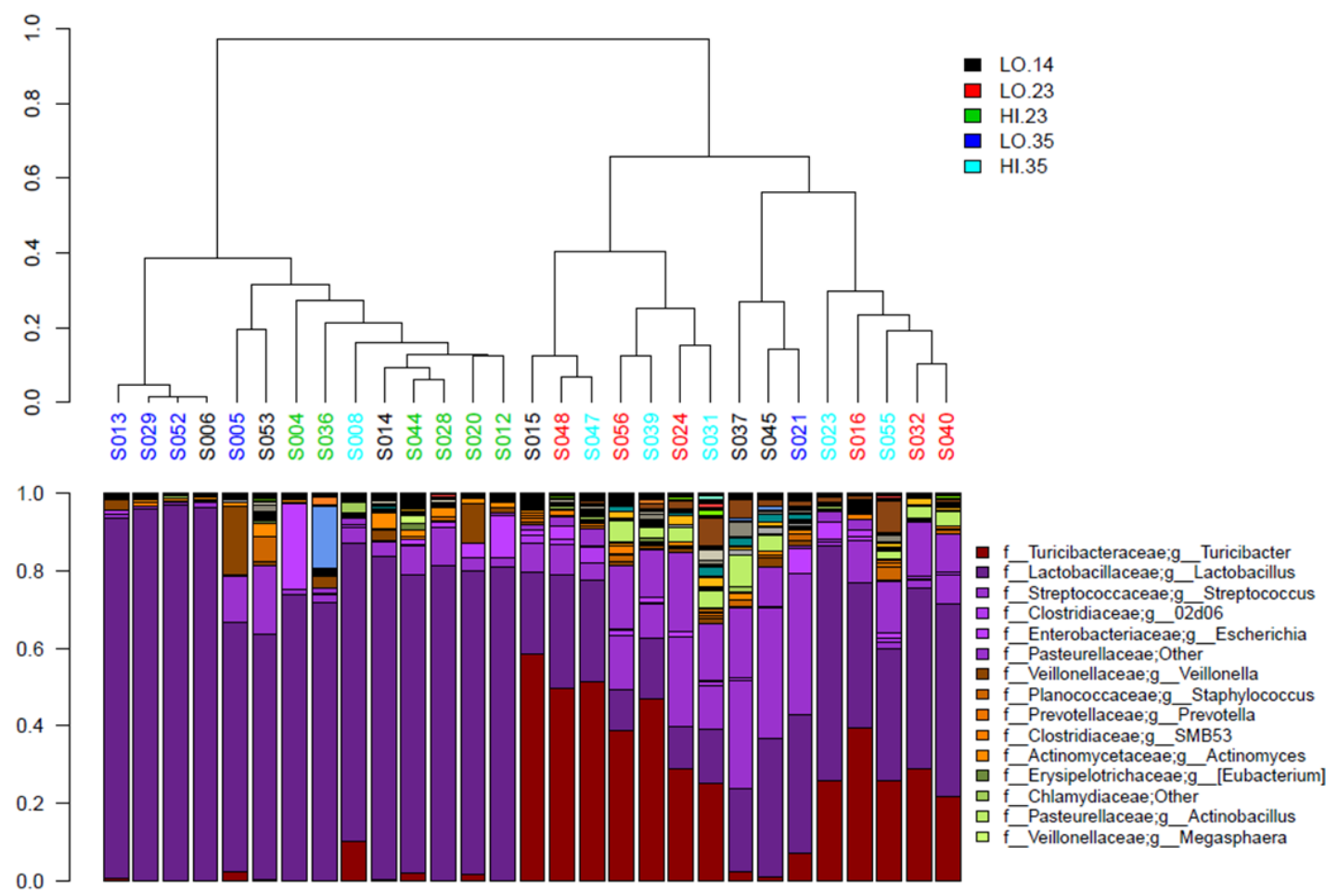

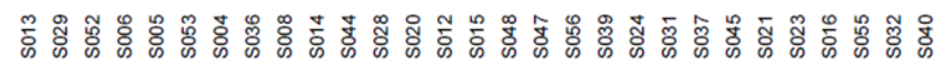

Figure 5. Cluster analysis of microbiota composition at genus level as analysed in ileum digesta and affected by treatment (control - LO; high zinc - $\mathrm{HI}$ ) and time point of measurement (d 14, 23 and 35). 
A further analysis of the microbiota composition in jejunal and ileal digesta was made by calculating different indices for microbiota diversity in the samples. In Figures 6 and 7, the data are provided on the a-diversity according to the Shannon index and the Chao 1 index, respectively. The diversity according to the Shannon index did not differ between time of life ( $d$ 14, 23 or 35) and experimental diet (either or not a high zinc diet from d 14-23) in both jejunal and the ileal digesta. Based on the Chao-1 index, however, significant effects on the ileal microbiota diversity of time (age of the piglets) $(P<0.001)$ and a significant age $x$ dietary treatment interaction $(P<0.01)$ was observed. The diversity was highest on d 35 and lowest on d 14, while on d 23 the diversity tended to be higher in piglets receiving the high zinc diet from d 14-23, compared to the diversity on $d 23$ of the animals receiving the control diet $(P<0.10)$. Based on the Chao- 1 index, no differences in the diversity were noticed with regard to the composition in jejunal digesta.
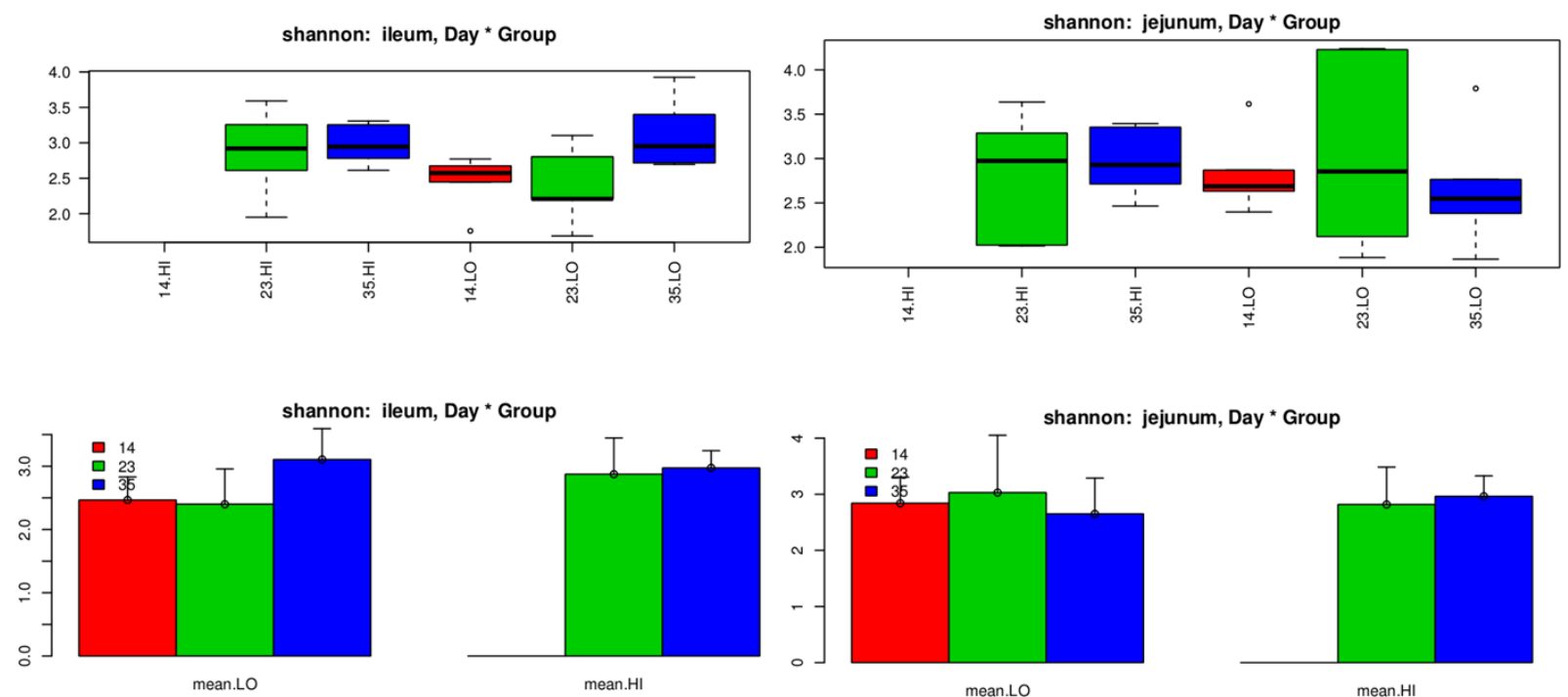

Figure 6. Shannon diversity on microbiota composition in the ileum (left panel) and the jejunum (right panel).
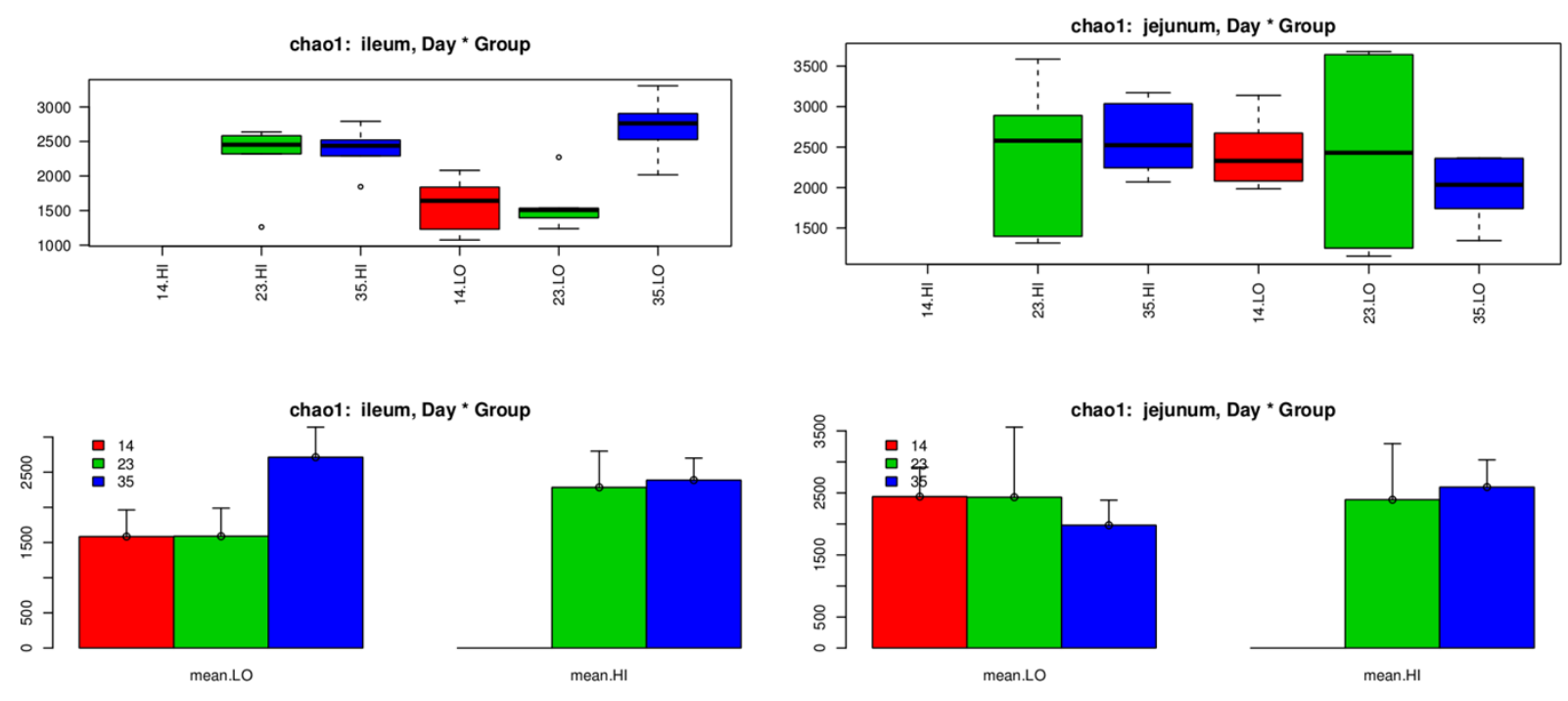

Figure 7. Chao 1 diversity on microbiota composition in the ileum (left panel) and the jejunum (right panel). 
In addition, the microbiota composition data were also analysed on the ß-diversity using a PCA Unifrac approach. The ß-diversity of microbiota composition relates to changes in diversity as effected by "environmental gradients" such as the intestinal location (jejunum and ileum) and dietary treatment $x$ time/age in the present study. It provides indications of changes in composition in terms of gain or loss of microbial species in a complex system. In Figure 8 an example is given to discriminate samples from the jejunum and the ileum in ß-diversity via Principal Component Analysis (PCA). The data in Figure 9 show that digesta samples of the jejunum and ileum can be discriminated rather well based on differences in ß-diversity, independent of age/time of sampling and dietary treatment.
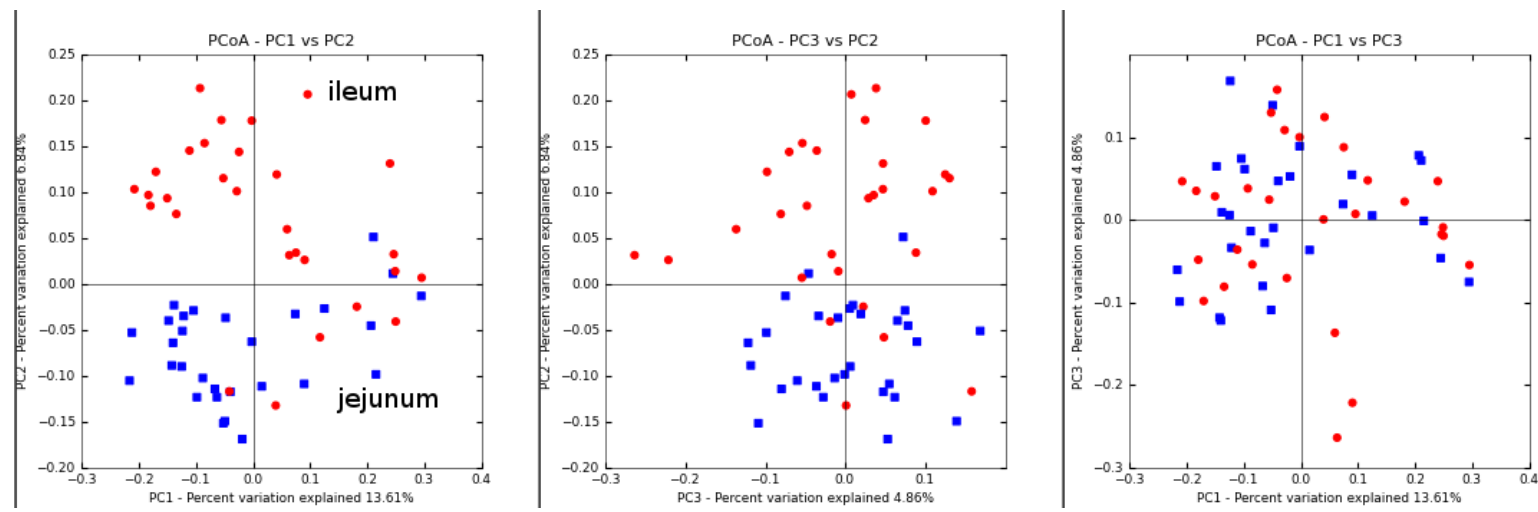

Figure 8. $\quad$ PCA plots of $\beta$ diversity based on unweighted Unifrac distances to discriminate between origin of samples (jejunal vs. ileal digesta samples).

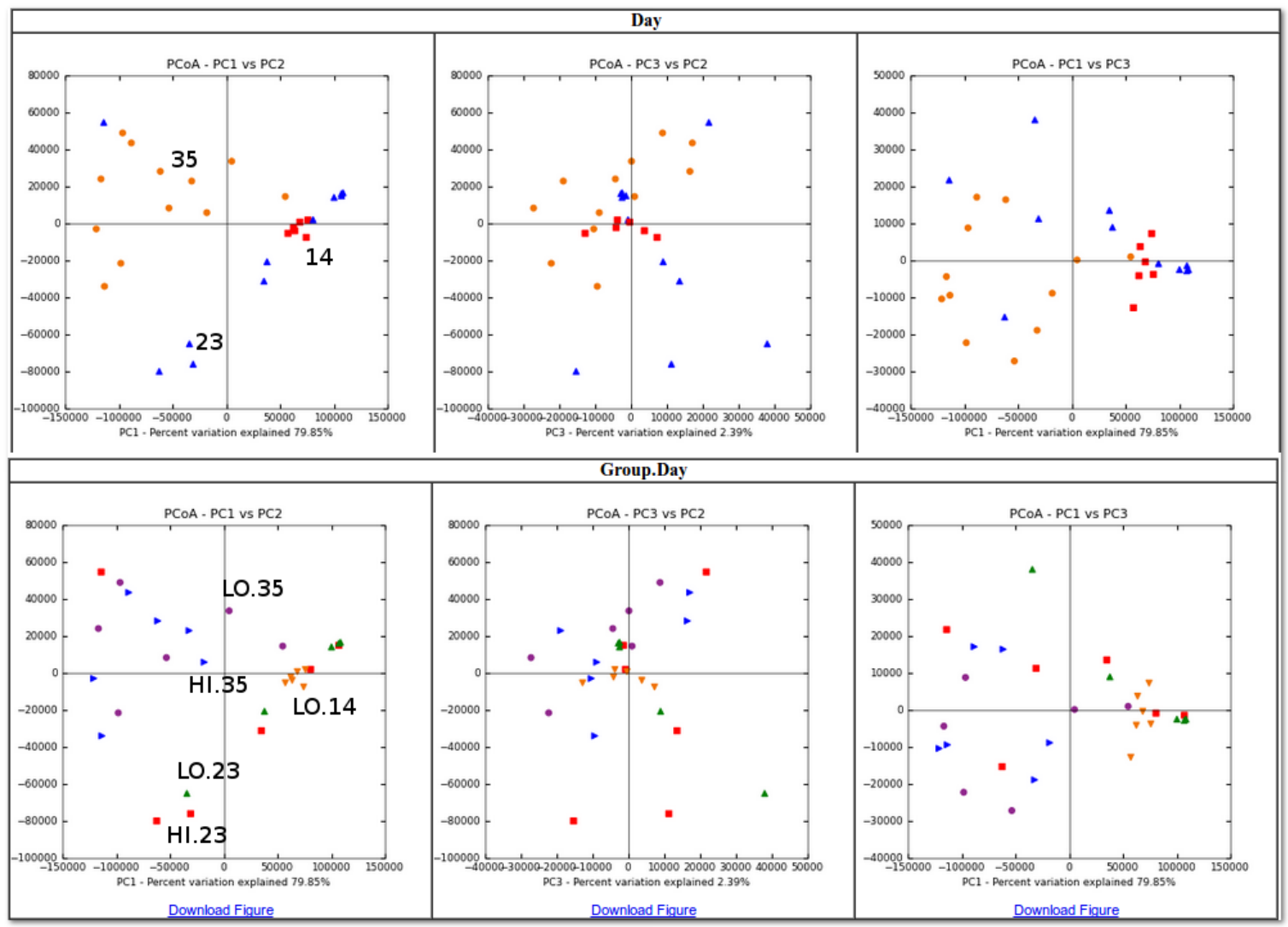

Figure 9. $\quad$ PCA plots of $\beta$ diversity based on taxonomic profile distances in ileal digesta to discriminate time/age of the animal (upper panel) and dietary treatment $x$ time/age (lower panel). 
PCA plots of $\beta$ diversity based on taxonomic profile distances as affected by age/time (day) and treatment (group) $x$ day show a rather good separation of samples based on these factors. This indicates that both age of the piglets and the dietary zinc intervention from d 14-23 had profound effects on the $\beta$ diversity of intestinal microbiota on both d 23 and 35 post weaning.

Finally, an ANOVA analysis was performed to evaluate whether there were differences in relative contribution at phylum, class, family, genus and species level of microbiota between samples obtained on d 14, d 23 (low or high Zn) or d 35 (low or high Zn). From family down to species level there were five to six taxa showing a difference in relative contribution at $\mathrm{P}<0.05$, with the constraint that their overall contribution was at least $1 \%$ of the total microbiota. An overview of the taxa concerned is given in Table 6 and further details are provided in Appendices 3 - 5.

Table 6. Overview of taxa at family, genus and species level showing a difference in relative contribution in jejunal digesta at $\mathrm{P}<0.05$ between dietary $\mathrm{Zn}$ treatment (group) $\mathrm{x}$ day (time/age), with the constraint that their overall contribution was at least $1 \%$ of the total microbiota.

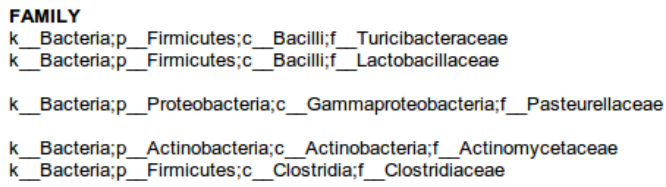

GENUS
SPECIES

Turicibacteraceae;g Turicibacters f Lactobacillaceae; L Lactobacillus;s __Lactobacillaceae; g_Lactobacillus;s__agilis Fasteurellaceae;Other; Other _Pasteurellaceae;g_Haemophilus;s parasuis f__Actinomycetaceae;g__Actinomyces;s_

\subsection{Gene expression in intestinal tissue}

To explore the whole genome expression data in intestinal mucosal tissue, including the effects of different time-points of sampling, dietary Zn treatments, and intestinal tissue location, a Principal Component Analysis was performed (Figure 10). This resulted in a clear distinction between samples of jejunal and ileal tissue, whereas the effects of time of sampling and dietary treatment were less clear. The results suggest a small time-treatment interaction effect at $d 23$ in both intestinal tissues, which is visualized by the open symbols deviating from the filled symbols in Figure 10. It should be mentioned that samples of two animals in the control group on d 23 (number 7768 (jejunum) and 7767 (ileum)) were considered as biological outliers and were therefore not removed from the dataset for subsequent analyses.

Differences in expression of specific genes as affected by dietary treatment (high zinc intervention) were explored on both time-points within a tissue ( $d 23$ and 35 in jejunum and ileum). Only comparisons made for $\mathrm{d} 23$ samples resulted in a number of genes being significantly expressed (Table 7). In intestinal tissue samples obtained on d 35 no differences in gene expression were observed in mucosal samples obtained from piglets that had received diets with a contrast in zinc concentration during the period of $d$ 14-23. Probes in samples of $d 23$ that were differently expressed and had an annotation were subsequently used as input for further functional analysis. 


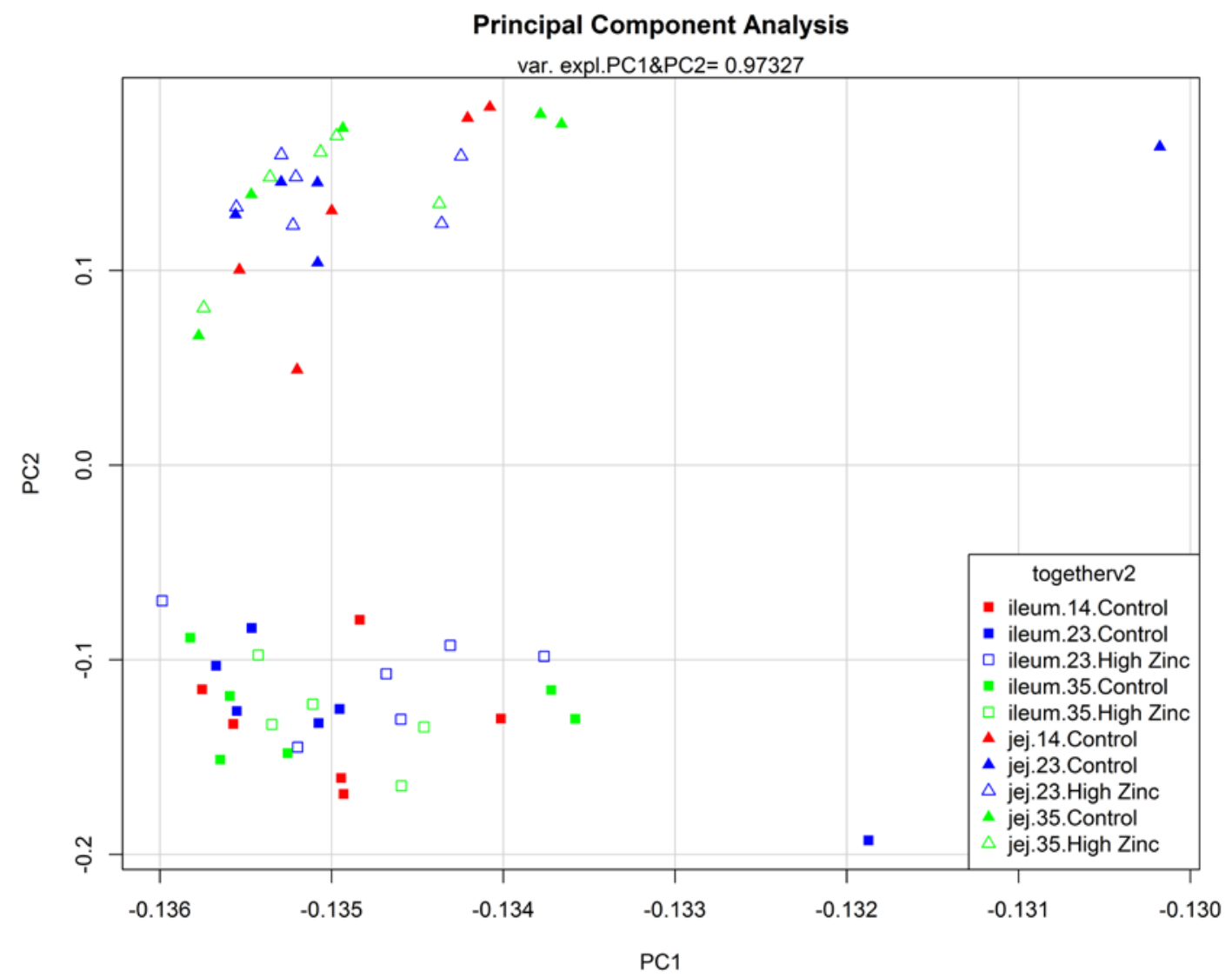

Figure 10. Principal Component Analysis of gene expression data of jejunal and ileal mucosal tissue of piglets of different treatments on $\mathrm{d} 14,23$, and 35 post weaning.

All arrays $(n=55)$ which passed the quality control are depicted. Tissue: jejunum (triangles) and ileum (boxes), Time: d 14 (red), 23 (blue), and 35 (green), and Treatment: control (filled symbols), high zinc (open symbols).

Table 7. Number of probes/genes up- or down regulated in the jejunal and ileal mucosa of piglets of the high zinc treatment as compared to the control treatment on $\mathrm{d} 23$.

\begin{tabular}{cccc} 
Tissue & & $U_{p}^{a}$ & Down \\
Jejunum & Probes & 11 & 13 \\
\hline \multirow{2}{*}{ Ileum } & Genes $^{\mathrm{b}}$ & 8 & 11 \\
\hline & Probes $^{\text {a }}$ & 22 & 10 \\
\hline Genes $^{\mathrm{b}}$ & 10 & 5 \\
\hline
\end{tabular}

\footnotetext{
${ }_{b}^{\mathrm{a}}$ Adjusted $\mathrm{P}$ value $<0.05$ and log Fold Change $>|1.5|$

Annotated genes
}

Functional analysis was performed by using two different web-based tools, being DAVID and Gene Decks. In DAVID it is possible to generate functional annotation clusters, in which different data repositories are queried and aggregated to 'functional clusters'. Table 8 shows a summary of the results of four separate runs using as input up- or down-regulated in jejunal and ileal mucosal tissue, respectively. Table 9 summarizes the results of a similar analysis by using the Gene Decks database. 
To further investigate whether whole gene sets were enriched based on the d 23 expression data, two additional separate analyses were performed for jejunal and ileal tissues. The former did not result in the identification of gene sets that were differentially expressed between dietary treatments considering the whole genome expression data of these samples ( $n=5$ or 6 ).

To further categorize the findings form the functional analysis and putting them into a more biological perspective, we followed a data mining strategy. In short, we used the differentially expressed genes as input for the STITCH database. In STITCH we added the first neighbours that interact with the genes/compounds. Subsequently, we extracted the given network and loaded this into Cytoscape, a software tool which allows for network reconstruction and visualization, in which we generated a new topology and identified the most frequent KEGG pathways for the genes in a particular network. This resulted in a network for both up- (Figure 11) and down-regulated genes (Figure 12).

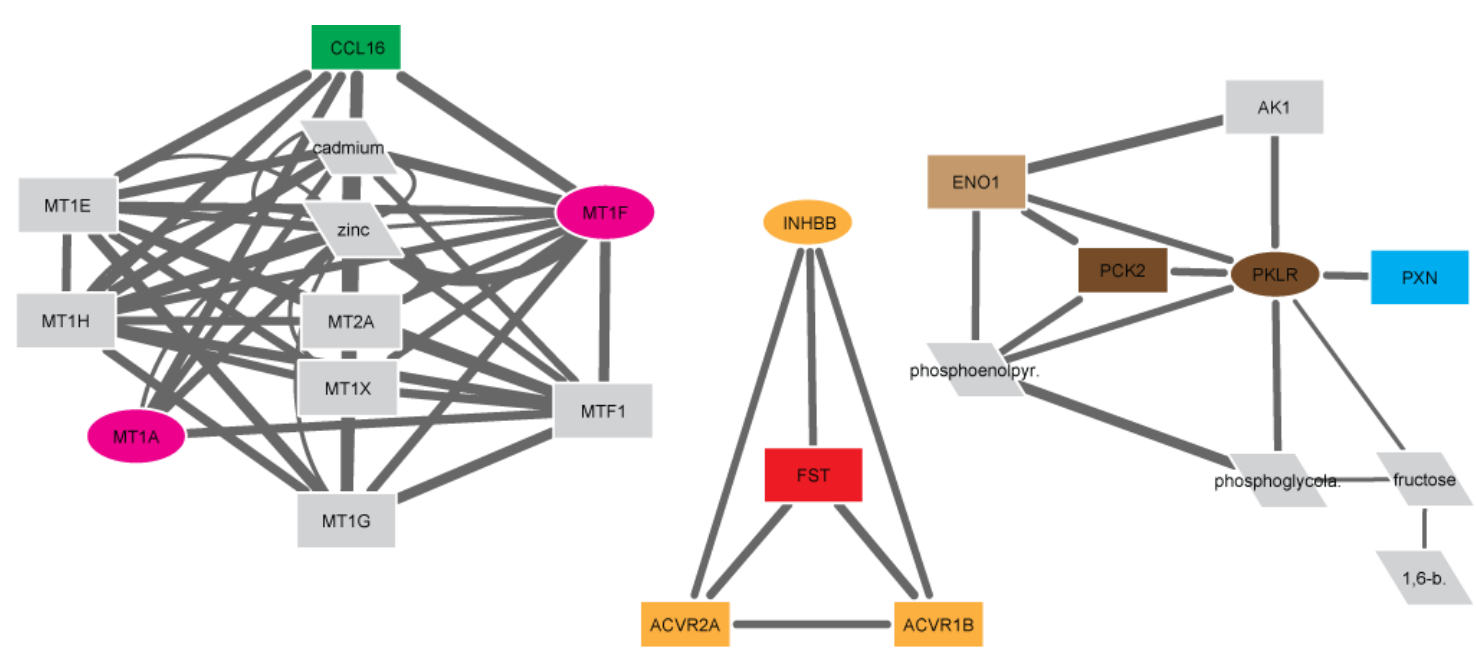

\section{Compound interaction partner STITCH}

Gene interaction partner STITCH

Regulated gene

TGFbeta signaling pathway

Glycolysis/Gluconeogenesis

TGFbeta signaling pathway + Cytokine-cytokine receptor interaction Glycolysis/Gluconeogenesis + Insulin signaling pathway Chemokine signaling pathway + Cytokine-cytokine receptor interaction

Mineral absorption

\section{Chemokine signaling pathway}

Figure 11. Network of up-regulated genes and their respective STITCH interaction partners (d 23). The seven most abundant KEGG pathways are highlighted. 


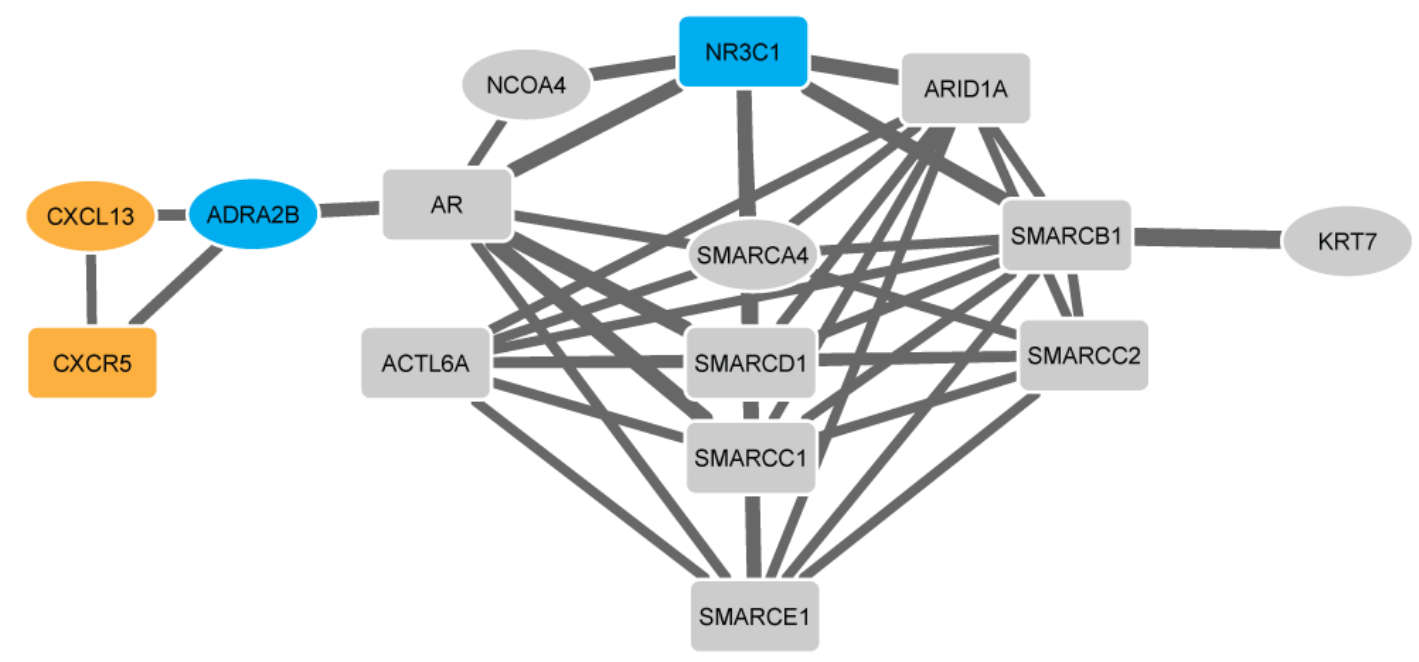

Regulated gene

STITCH interaction partner
Chemokine signaling pathway + Cytokine-cytokine interaction

Neuroactive ligand-receptor interaction

Figure 12. Network of down-regulated genes and their respective STITCH interaction partners (d 23). The three most abundant KEGG pathways are highlighted, i.e. 'chemokine signalling pathway', 'cytokine-cytokine-interactions', and 'neuroactive ligand-receptor interaction'.

Figure 11 shows that a number of upregulated genes in the jejunal mucosa on $d 23$ by the high zinc intervention from d 14-23 are involved in pathways related to mineral absorption, immune signalling and cell energy metabolism (glycolysis and gluconeogenesis). A few down regulated genes by the $\mathrm{Zn}$ intervention are also involved in immune signalling pathways (Figure 12). 
Table 8. Number of gene sets up- or down regulated in the jejunal and ileal mucosa of piglets of the high zinc treatment as compared to the control dietary treatment on d 23 as determined via DAVID Functional Annotation Clustering.

\begin{tabular}{|c|c|c|c|c|c|c|}
\hline Tissue & $\begin{array}{l}\text { Regu- } \\
\text { lation }\end{array}$ & $\begin{array}{l}\text { \# of } \\
\text { clusters }\end{array}$ & Generalized term & $\begin{array}{l}\text { \# of } \\
\text { genes }\end{array}$ & $\mathrm{ES}^{\mathrm{a}}$ & Genes \\
\hline \multirow[t]{6}{*}{ Jejunum } & Up & 1 & metal ion binding & 3 & 1.35 & MT1A ${ }^{b}, M T 1 F^{b}, \operatorname{COX}^{b}$ \\
\hline & \multirow[t]{5}{*}{ Down } & \multirow[t]{5}{*}{5} & signal peptide & 5 & 0.93 & 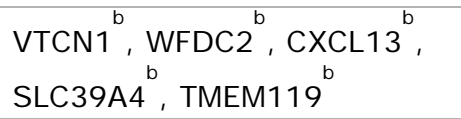 \\
\hline & & & disulfide bond & 4 & 0.73 & $\begin{array}{l}\text { VTCN1 }{ }^{b}, \text { WFDC2 }{ }^{b}, \text { ADRA2B } \\
\text { CXCL13 }\end{array}$ \\
\hline & & & (trans) membrane & 4 & 0.42 & $\begin{array}{l}\text { VTCN1, ADRA2B, SLC39A4, } \\
\text { TMEM119 }\end{array}$ \\
\hline & & & glycoprotein / splicing & 4 & 0.24 & $\begin{array}{l}\text { VTCN1, WFDC2, ADRA2B, } \\
\text { SLC39A4 }\end{array}$ \\
\hline & & & $\begin{array}{l}\text { phosphoprotein / } \\
\text { polymorphism }\end{array}$ & 6 & 0.13 & $\begin{array}{l}\text { SMARCA } 4^{\mathrm{b}}, \mathrm{KRT7}^{\mathrm{b}}, \mathrm{NCOA}^{\mathrm{b}}{ }^{\prime} \\
\text { ADRA2B, SLC39A4, TMEM119 }\end{array}$ \\
\hline \multirow[t]{2}{*}{ Ileum } & Up & 1 & metal ion binding & 4 & 0.75 & $\begin{array}{l}\text { MT1A }{ }^{b}, M T 1 F^{b}, \text { SLC39A } 4^{b} \\
\text { PKLR }\end{array}$ \\
\hline & Down & 0 & & 0 & & \\
\hline
\end{tabular}

a avgES; Enrichment Score [i.e -log(average(P-value descriptors)]

${ }^{b}$ interacting in STITCH networks 
Table 9. Number of gene sets up- or down regulated in the jejunal and ileal mucosa of piglets of the high zinc treatment as compared to the control dietary treatment on $\mathrm{d} 23$ as determined by Gene Decks analysis.

\begin{tabular}{|c|c|c|c|c|c|c|}
\hline Tissue & $\begin{array}{l}\text { Regula- } \\
\text { tion }\end{array}$ & $\begin{array}{c}\text { \# of } \\
\text { descriptors }\end{array}$ & Generalized term & $\begin{array}{l}\text { \# of } \\
\text { genes }\end{array}$ & $\mathrm{ES}^{\mathrm{a}}$ & Genes \\
\hline \multirow[t]{4}{*}{ Jejunum } & Up & 17 & metalthion & 2 & 3.84 & $M T 1 A^{b}, M T 1 F^{b}$ \\
\hline & \multirow[t]{3}{*}{ Down } & 19 & carcinoma & 4 & 3.62 & $\begin{array}{l}\text { KRT7 }^{\mathrm{b}}, \mathrm{NCOA}^{\mathrm{b}}, \\
\text { SMARCA } 4^{\mathrm{b}}, \text { WFDC2 }\end{array}$ \\
\hline & & 9 & transcription / hormone & 2 & 3.3 & NCOA4 ${ }^{b}$, SMARCA $4^{b}$ \\
\hline & & 8 & cancer & 6 & 3.55 & $\begin{array}{l}\text { ADRA2B }^{\mathrm{b}}, \mathrm{CXCL}^{\mathrm{b}} 3^{\mathrm{b}}, \\
\text { KRT7 }^{\mathrm{b}},{ }^{\mathrm{b}} \text {, SMARCA4 } \\
\text { VTCN1 }^{\mathrm{b}}, \text { SLC39A4 }\end{array}$ \\
\hline \multirow[t]{3}{*}{ Ileum } & \multirow[t]{2}{*}{ Up } & 17 & metalthion & 2 & 3.84 & MT1A ${ }^{b}, M_{1 F}^{b}$ \\
\hline & & 3 & insulin/immune & 2 & 2.5 & INHBB, PKLR \\
\hline & Down & 9 & $\begin{array}{c}\text { disease } \\
\text { (including gut) }\end{array}$ & 3 & 3.01 & BPI, DMBT1, REG3G \\
\hline
\end{tabular}

a avgES; Enrichment Score [i.e -log(average(P-value descriptors)]

${ }^{b}$ interacting in STITCH networks 


\section{Discussion}

The present study was carried out to evaluate whether provision of a high zinc diet for a short period of time after the immediate post-weaning phase of piglets ( $d 4$ till 23 post weaning) as a "model intervention" would influence intestinal microbiota composition and intestinal mucosal gene expression at the end of the dietary intervention ( $d 23$ post weaning) and twelve days after the termination of the intervention ( $d 35$ post weaning). The study should provide information on whether a short high zinc dietary intervention as model intervention could potentially interfere with the development of immune competence in pigs. It is well known that intestinal microbiota composition and activity interferes with the development and functionality of the local immune system in the gut in different animal species and in man (Round \& Mazmanian, 2009; Hooper et al., 2012). Diet ingredient composition and inclusion of particular functional ingredients, such as pre- and probiotics and substances and extracts of plant origin with antimicrobial properties, can modulate the intestinal microbiota composition in various compartments of the intestinal tract (van Krimpen et al., 2014; Haenen et al., 2013; Taras et al., 2007; Jensen, 1998). There is an intimate contact between the luminal and gut wall associated microbiota and the intestinal mucosa. This interplay indicates that changes in intestinal microbiota composition can also influence responses of gut tissue towards these changes, including changes of responses of the immune system present in the mucosa, submucosa and lamina propria of the gut. It is estimated that a major part of the immune cells present in the body are located in intestinal tissues. Therefore, a change of the intestinal microbiota could also interfere with delicate crosstalk between the intestinal microbiota and intestinal tissue of the host. Given the diversity of inflammatory or immune responses that can be mounted by the intestinal epithelium, association of e.g. probiotics with epithelial cells might be sufficient to trigger signalling pathways that ultimately activate underlying immune cells in the lamina propria. Alternatively, probiotics or other dietary constituents may also release or contain factors that themselves trigger signalling cascades at the level of the epithelium or associated immune system (Corthésy et al., 2007).

Various studies have highlighted how the microbiota elicit innate and adaptive immune mechanisms that cooperate to protect the host and maintain intestinal homeostasis. Epithelial cells are a central component of the immune system of the gut. In a similar manner to immune cells, epithelial cells express receptors for microbial-associated molecular patterns (MAMPs). These receptors activate signalling cascades that finely tune epithelial cell production of antimicrobial products and chemokines, depending on the signals that are delivered by intestinal microbiota (Cerf-Bensussan \& GaboriauRouthiau, 2010). Thus, gut epithelial cells form a potent and inducible physico-chemical barrier, which limits microbial growth and access to the gut surface. They can also recruit leukocytes to complement their barrier function or to participate in the activation of gut adaptive immune responses. In mammals, the development of gut-associated lymphoid tissues (GALT) is initiated before birth by a genetic programme. However, GALT maturation and the recruitment of IgA-secreting plasma cells and activated T cells to mucosal sites only occurs after birth and is strictly dependent on microbiotaderived signals. These signals influence the crosstalk between epithelial cells and gut dendritic cells (DCs), thereby modulating the nature and intensity of intestinal B and T cell responses (CerfBensussan \& Gaboriau-Routhiau, 2010).

Given the former, dietary constituents and specific interventions, such as a high concentration of dietary zinc in the present study, could exert both direct and indirect effects on the intestinal mucosa and its residing immune system. Therefore, dietary interventions at young age could interfere with the development of immune competence and responsiveness of the immune system at later age.

The present study showed that a short term intervention with a high zinc diet $(2500 \mathrm{mg} / \mathrm{kg}) \mathrm{using}$ $\mathrm{ZnO}$ as source of zinc, had a significant influence on microbiota composition and diversity of small intestinal digesta, in particular in the ileum. On d 35, twelve days after feeding the control diet with a regular $\mathrm{Zn}$ concentration $(100 \mathrm{mg} / \mathrm{kg}$ ) similar to the control group, the composition and diversity was 
still different compared to the control group. Analysis of differences in microbiota composition at phylum, class, family, genus and species level only revealed statistical differences in relative contribution in ileal digesta for a few taxa. The latter may be related to the relatively low number of samples analysed per experimental treatment and to other factors inducing variation in intestinal microbiota composition between animals, including genetic differences.

In addition, it was observed that microbiota composition in both treatment groups changed in composition and diversity in time over a period of d 14 till 35 post weaning. The former likely relates to the gradual succession of microbial species and associated maturation of the complex intestinal microbiota during the first period of life.

Pieper et al. (2012) also observed effects of dietary zinc oxide concentrations (50, 150, 250, 1000 and $2500 \mathrm{mg} \mathrm{zinc} / \mathrm{kg}$ ) on microbiota composition in ileal digesta of piglets over a period of two weeks (one to three weeks after weaning). Ileal bacterial community profiles were analysed by denaturing gradient gel electrophoresis and selected bacterial groups quantified by real-time PCR. Species richness, Shannon diversity and evenness were significantly higher at high ZnO levels. Quantitative PCR revealed lowest total bacterial counts in the $50 \mathrm{mg} / \mathrm{kg}$ group. Increasing ZnO levels led to an increase in Enterobacteria from log 4.0 CFU/g digesta $(50 \mathrm{mg} / \mathrm{kg}$ ) to log $6.7 \mathrm{CFU} / \mathrm{g}$ digesta (2500 $\mathrm{mg} / \mathrm{kg}$ ). The increase in microbiota diversity in the gut by a high zinc intervention is in line with observations in the present study.

It is well established that dietary zinc oxide at pharmacological level helps to prevent post-weaning diarrhoea and improve pig performance, but the underlying mechanisms are still not clear. Besides the influence of high zinc doses on digestive function, immune system, muscle growth, transcription, apoptosis and hormone status, changes in intestinal microbial community composition could explain the reduction in diarrhoea in piglets (Suttle, 2010). In fact, the results presented by Liu et al. (2014) and the present study support previous findings showing that some bacterial groups are suppressed by high levels of dietary zinc oxide, whereas others increase in abundance at high zinc doses (Katouli et al., 1999; Højberg et al., 2005; Vahjen et al., 2010). An increased bacterial diversity in the digestive tract could be an indicator for a more stable bacterial ecosystem, thus resisting perturbations that could lead to impaired function and health of the gut.

The present study also revealed effects of the high zinc intervention on gene expression in the jejunal and ileal mucosa, in particular on d 23, at the end of the intervention period.

No effects on intestinal gene expression were observed on d 35 post weaning, twelve days after termination of the dietary intervention. Both up and down regulation of genes was observed as affected by the dietary $\mathrm{Zn}$ intervention. The changes in gene expression were in part related to genes (e.g. MT1A and MT1F) encoding for the synthesis of metallothioneins,

involving mineral absorption, intracellular transport and regulation of intracellular mineral concentrations. Metallothionein has been shown to control the amount of zinc available in cells and participates in the transfer of essential metal ions to critical stress response proteins and transcription factors such as zinc-finger proteins, apoenzymes and other metalloproteins (Wintergerst et al., 2007). In line, also the expression of SLC39A4 was affected in the jejunal and ileal mucosa in the present study. This gene encodes for a zinc transporter and plays an important role in cellular zinc homeostasis. The former observations seem to be directly related to the contrast in intestinal supply of $\mathrm{Zn}$ via the diet in the present study.

At both the jejunal and the ileal level, the expression of the gene encoding for metallothioneins (MT) was increased. MT are small, cysteine-rich proteins with also significant immune modulatory activity. They have been shown to play a critical role in important cellular mechanisms including heavy metal detoxification, essential metal management and inflammatory responses. MT production can be induced by a number of cellular stressors and acts to lessen the harmful effects of oxidizing agents and heavy metal exposure such as zinc (Roy- O'Reilly, 2012). There is evidence in humans that the level of metallothioneins may have significant effects on the adaptive immune response. Roy-O'Reilly (2012) showed in research with mice that variation in gene expression for MT affects the speed and degree of the lymphocyte proliferative response. It has been hypothesized that the lack of functional 
MT reduces the ability to handle elevated levels of reactive oxygen species (ROS). This increase in intracellular ROS leads to activation of transcription factors like NF-kB, which augments the expression of immunomodulatory cytokines (Crowthers et al., 2000). A link between the expression of genes encoding for metallothionein $1 A$ and IL- 6 was observed in airway tissue of sheep stressed by physical injury (Yahaya et al., 2013). In essence, a lack of endogenous MT creates a primed immune response that could potentially lead to autoimmune reactions in man under stressful conditions. Metallothioneins have also been found to be a essential component of the pathogen induced immune response, in particular in the early innate immune response to a Listeria infection in mice. Both mice with increased levels of MT and mice with decreased levels of MT were shown to be more resistant to early Listeria infection (Emeny et al., 2009). It has also been suggested that under inflammatory conditions, metallothioneins in the extracellular environment may support the movement of leukocytes to the site of inflammation (Yin et al., 2005). In pigs, zinc supplementation was associated with a marked decrease in expression of immune response genes concerned with inflammation, possibly related to the stage of infection (Sargeant et al., 2010). The key finding from their study was the differential expression of a number of genes associated with immunity and inflammation between dietary treatments. In the intestinal mucosa, expression of e.g. Peptidase Inhibitor 3 (PI3) (a gene that encodes an elastase-specific inhibitor that functions as an antimicrobial peptide against Gram-positive and Gram-negative bacteria), Bactericidal/Permeability-Increasing Protein (BPI) (a gene encoding a lipopolysaccharide binding protein) and Dual Oxidase 2 (DUOX2), each encoding antimicrobial molecules, and PSPD, encoding an opsonin, being a molecule that enhances phagocytosis, was lower in piglets fed a diet with supplemented with ZnO. They concluded that ZnO supplementation in post weaning ETEC challenged piglets may improve piglet performance, at least in part, by decreasing the level of intestinal inflammation caused by ETEC challenge, reducing disruption of normal intestinal morphology and function. Zinc oxide may also influence the severity of infection through altered expression of pathogen receptors (Sargeant et al., 2010). In our study, also expression of the CXCL13 gene was affected in jejunal mucosal tissue by the $\mathrm{Zn}$ intervention. This gene codes for a protein that has chemotactic properties for B-lymphocytes, but not for T-lymphocytes, monocytes and neutrophils and promotes homing

B-cells from the periphery to Peyer's patches. This shows another link between the zinc intervention and changes in responses of the immune system. Some other genes in intestinal mucosal tissues were up- or down regulated by the dietary zinc intervention in the present study, which were in part also related to pathways involved in the immune system (e.g. signalling pathways and cytokine receptor interactions). The further biological consequences of these changes, in particular for the functioning of the immune system of pigs are not clear yet. The present data do also not reveal whether these effects are a direct effect related to the differences in dietary zinc supply and absorption or are indirect effects related to changes in the intestinal microbiota composition as induced by dietary supplementation of $\mathrm{ZnO}$ at pharmaceutical levels.

Zhang and Guo (2009) evaluated the pharmacological effect of Zn (2000 mg Zn/kg) on occurrence of diarrhoea in relation to intestinal permeability of post weaning piglets. Expression of mucosal tight junction protein was measured at RNA and protein level. Inclusion of tetrabasic zinc chloride (TBZC) or ZnO in the diet significantly increased average daily gain and average daily feed intake, while leading to reduced feed conversion ratio and faecal scores. TBZC reduced urinary lactulose : mannitol ratios of weaning piglets, while dietary supplementation with ZnO tended to reduce urinary lactulose : mannitol ratios. ZnO or TBZC significantly enhanced the mRNA and protein expression of occludin and zonula occludens protein-1 (ZO-1) as important tight junction proteins in the ileal mucosa. Piglets fed the TBZC-supplemented diet had a higher level of occludin than pigs fed the ZnO-supplemented diet. The results indicates that $\mathrm{Zn}$ supplementation decreased faecal scores and the reduction was accompanied by reduced intestinal permeability, which was evident from the reduced urinary lactulose : mannitol ratios and increased expression of occludin and ZO- 1 . Therefore, the protective effect of pharmacological levels of dietary $\mathrm{Zn}$ in reducing diarrhoea might, at least partly, also be associated with a reduced intestinal permeability.

Liu et al. (2014a) studied the effects of increasing levels of dietary Zn on mRNA expression levels of mucin (MUC) 1, 2, 13, 20, toll-like receptor (TLR) 2, 4, interleukin (IL)-1B, 8, 10, interferon-c (IFN- $\mathrm{Y}$ ) and transforming growth factor- $ß$ (TGF-ß) in the colon of piglets. The colonic crypt area increased in 
an age-depending manner, and the greatest area was found with medium concentration of dietary zinc. With the high concentration of dietary zinc, the number of goblet cells containing mixed neutralacidic mucins and total mucins increased. The mRNA expression of TLR4 and the pro-inflammatory cytokine IL-8 were down-regulated with high dietary zinc treatment, while piglets fed with medium dietary zinc had the highest expression. It was concluded that dietary zinc level had a clear impact on colonic morphology, mucin profiles and immunological traits in piglets after weaning. These changes might support local defence mechanisms and affect colonic physiology and contribute to the reported reduction of post-weaning diarrhoea. Similarly, in our study the expression of INHBB was affected by the $\mathrm{Zn}$ intervention. INHBB codes for inhibins/activins involved in regulating a number of diverse functions such as hypothalamic and pituitary hormone secretion, gonadal hormone secretion, germ cell development and maturation, erythroid differentiation, insulin secretion, nerve cell survival, embryonic axial development or bone growth, depending on their subunit composition. Inhibins appear to oppose the functions of activins. The link between the dietary zinc treatment and the energy metabolism at cellular level in the intestinal mucosa (expression of INHBB and PKLR, the protein encoded by this gene is a pyruvate kinase that catalyses the transphosphorylation of phohsphoenolpyruvate into pyruvate and ATP, which is the rate-limiting step of glycolysis) and network analysis in Figure 11 could be related to a shift in energy metabolism from aerobe to anaerobe metabolism in cells exposed to high $\mathrm{Zn}$ conditions and to high intracellular oxidant concentrations (i.c. Zn).

Liu et al. (2014b) studied the effects of different dietary Zn concentrations in post weaning piglets on intestinal mucosal morphology and the number of goblet cells producing neutral, acidic, sulfated, and sialylated mucins, intraepithelial lymphocytes and gene expression of mucin 2 (MUC2), mucin 20 (MUC20), ß-defensin 3, and trefoil factor 3 (TFF3). Villus height and crypt depth were not affected by dietary $\mathrm{Zn}$ concentration. The mucin types were modified mainly by age, and dietary $\mathrm{Zn}$ had no effect in the proximal jejunum. In the distal jejunum, $Z n$ concentration had effects on the mucin types found. A high dietary $\mathrm{ZnO}$ reduced the sulfomucins and increased the sialomucins in the crypts. High dietary $Z n$ treatment led to a reduced abundance of CD8+ y $\delta T$-cells. The expression of MUC2 and MUC20 was not influenced by age or dietary Zn concentration. A high Zn intake resulted in a reduced gene expression of $ß$-defensin 3, but did not affect the expression of TFF3. It was concluded that dietary $\mathrm{Zn}$ concentration supplied as $\mathrm{ZnO}$ has specific effects on the innate and adaptive gut associated immune system of piglets.

It can be concluded from the present study that provision of a diet with a high concentration of zinc as zinc oxide during a short period of time ( 9 days) to piglets in the post weaning period ( $d$ 14-23 post weaning) induces differences intestinal microbiota composition in particular in the ileum and on the intestinal expression of genes in part related to the functioning of the local innate immune system. The high dietary zinc intervention can therefore be considered as a suitable model for studying relationships between dietary interventions, intestinal microbiota composition and development of immune competence in post weaning piglets. Introducing the intervention more closely to the moment of weaning might increase the size of effects of the model intervention as the intestinal microbiota is less established and less stable shortly after weaning. 


\section{$5 \quad$ References}

Camilleri, M., M., Madsen, K., Spiller, R., Van Meerveld, B. G., \& Verne, G. N. (2012). Intestinal barrier function in health and gastrointestinal disease. Neurogastroenterol Motil. 24, 503-512.

Cerf-Bensussan, N., \& Gaboriau-Routhiau, V. (2010). The immune system and the gut microbiota: friends or foes?. Nature Reviews Immunology, 10, 735-744.

Corthésy, B., Gaskins, H. R., \& Mercenier, A. (2007). Cross-talk between probiotic bacteria and the host immune system. The Journal of Nutrition, 137, 781S-790S.

Crowthers, K. C., Kline, V., Giardina, C., \& Lynes, M. A. (2000). Augmented humoral immune function in metallothionein null mice. Toxicol. Appl. Pharmacol., 166, 161-172.

CVB (2011a). Tabellenboek Veevoeding (2011). Centraal Veevoederbureau (CVB), The Netherlands. CVB-reeks nr 46. In Dutch.

CVB (2011b). Veevoedertabel 2011. Data on the chemical composition, digestibility and nutritional value of feedstuffs. Centraal Veevoederbureau (CVB), The Netherlands. In Dutch.

Emeny, R.T., Emeny, R. T., Marusov, G., Lawrence, D. A., Pederson-Lane, J., Yin, X., \& Lynes, M. A. (2009). Manipulations of metallothionein gene dose accelerate the response to Listeria monocytogenes. Chem. Biol. Interact., 181, 243-1253.

Grimble R.F. (2001). Nutritional modulation of immune function. Proc Nutr Soc. 60:389-97.

Gruys E., Toussaint M.J., Niewold T.A., Koopmans S.J., van Dijk, E., Meloen R.H. (2006). Monitoring health by values of acute phase proteins. Acta Histochem., 108, 229-232.

Haenen, D., Zhang, J., da Silva, C. S., Bosch, G., van der Meer, I. M., van Arkel, J., J.G.C. van den Borne and Hooiveld, G. J. (2013). A diet high in resistant starch modulates microbiota composition, SCFA concentrations, and gene expression in pig intestine. The J ournal of Nutrition, 143, 274-283.

Hojberg O., N. Canibe, H.D. Poulsen, M.S. Hedemann,B.B. Jensen (2005). Influence of dietary zinc oxide and copper sulfate on the gastrointestinal ecosystem in newly weaned piglets. Appl. Environ. Microbiol., 71, 267-2277.

Hooper, L. V., Littman, D. R., \& Macpherson, A. J. (2012). Interactions between the microbiota and the immune system. Science, 336, 1268-1273.

Jensen, B. B. (1998). The impact of feed additives on the microbial ecology of the gut in young pigs. Journal of Animal and Feed Sciences, 7, 45-64.

Kelly, D., T. King, and R. Aminov (2007). Importance of microbial colonization of the gut in early life to the development of immunity. Mutat. Res., 622, 58-69.

Liu, P., Pieper, R., Rieger, J., Vahjen, W., Davin, R., Plendl, J., Meyer, W., Zentek, J. (2014a). Effect of dietary zinc oxide on morphological characteristics, mucin composition and gene expression in the colon of weaned piglets. PloS One, 9(3), e91091.

Liu, P., Pieper, R., Tedin, L., Martin, L., Meyer, W., Rieger, J., Plendl J., Vahjen W. and J. Zentek (2014b). Effect of dietary zinc oxide on jejunal morphological and immunological characteristics in weaned piglets. Journal of Animal Science, 92, 5009-5018.

Martin, L. R. Pieper, N. Schunter, W. Vahjen, J. Zentek (2013). Performance, organ zinc concentration, jejunal brush border membrane enzyme activities and mRNA expression in piglets fed with different levels of dietary zinc. Arch. Animal Nutr., 67, 248-261.

Payne, R.W., P.W. Lane, A.E. Ainsley, K.E. Bicknell, P.G.N. Digby, S.A. Harding, P.K. Leech, H.R. Simpson, A.D. Todd, P.J. Verrier, R.P. White (1993). Genstat 5. Reference Manual. Oxford University Press, Oxford, UK.

Penders J, Thijs C, Vink C, Stelma FF, Snijders B, et al. (2006). Factors influencing the composition of the intestinal microbiota in early infancy. Pediatrics 118: 511-521.

Peterson, L.W. and D. Artis (2014). Intestinal epithelial cells: regulators of barrier function and immune homeostasis. Nat. Rev. I mmunol., 14, 141-53.

Round, J. L., \& Mazmanian, S. K. (2009). The gut microbiota shapes intestinal immune responses during health and disease. Nature Reviews Immunology, 9, 313-323. 
Roy-O-Reilly, M. (2012). Metallothionein Gene Dose and the Immune Response. Honors Thesis. University of Connecticut, US.

Sargeant, H.R., McDowall, K.J., Miller, H.M., Shaw, M.A. (2010). Dietary zinc oxideaffects the expression of genes associated with inflammation: transcriptome analysis in piglets challenged with ETEC K88. Vet. Immunol. I mmunopathol. 137, 120-129.

Schokker, D., Zhang, J., Zhang, L.L., Vastenhouw, S.A., Heilig, H.G., Smidt, H., Rebel, J.M. and Smits, M.A. (2014). Early-life environmental variation affects intestinal microbiota and immune development in new-born piglets. PLoS One, 9(6): p. e100040.

Skrovanek, S., DiGuilio, K., Bailey, R., Huntington, W., Urbas, R., Mayilvaganan, B., G. Mercogliano \& J.M. Mullin, J. M. (2014). Zinc and gastrointestinal disease. World Journal of Gastrointestinal Pathophysiology, 5, 496.

Snedecor, G.W., W.G. Cochran (1980). Statistical Methods. 7th ed. I owa State University Press, Ames, USA.

Taras, D., Vahjen, W., \& Simon, O. (2007). Probiotics in pigs - modulation of their intestinal distribution and of their impact on health and performance. Livestock Science, 108, 229-231.

Turner, J.R. (2009). Intestinal mucosal barrier function in health and disease. Nat. Rev. Immunol., 9, 799-809.

Vahjen W., Pieper R., Zentek J. (2011). Increased dietary zinc oxide changes the bacterial core and enterobacterial composition in the ileum of piglets. J. Anim. Sci., 89, 2430-2439.

Vahjen, W., Pieper, R., Zentek, J., (2010). Bar-coded pyrosequencing of 16S rRNA gene amplicons reveals changes in ileal porcine bacterial communities due to high dietary zinc intake. Appl. Environ. Microbiol. 76, 6689-6691.

Van Krimpen, M.M., M.M. Hulst, J. van der Meulen, D. Schokker, H.F.J . savelkoul, E.J . Tijhaar, V.P.M.G. Rutten (2014). Nutritional intervention in animals: benchmarking of strategies, monitoring biomarkers and immune competence. Report Wageningen UR Livestock Research Nr. 800.

Wang X., Ou D., Yin J., Wu, G., Wang J. (2009) Proteomic analysis reveals altered expression of proteins related to glutathione metabolism and apoptosis in the small intestine of zinc oxidesupplemented piglets. Amino Acids, 37, 209-218.

Wintergerst, E.S., S. Maggini, and D.H. Hornig, (2007). Contribution of selected vitamins and trace elements to immune function. Ann. Nutr. Metab., 51, 301-323.

Yahaya, B., McLachlan, G., McCorquodale, C., \& Collie, D. (2013). Gene Expression Changes Associated with the Airway Wall Response to Injury. PloS One, 8 (4), e58930.

Yin, X., Knecht, D. A., \& Lynes, M. A. (2005). Metallothionein mediates leukocyte chemotaxis. BMC immunology, 6, 21. DOI: 10.1186/1471-2172-6-21.

Zhang, B., \& Guo, Y. (2009). Supplemental zinc reduced intestinal permeability by enhancing occludin and zonula occludens protein-1 (ZO-1) expression in weaning piglets. British Journal Nutrition, 102, 687-693. 


\section{Appendices}

Appendix 1. Overview of microbiota composition at genus level in jejunal digesta as affected by age (time) and dietary treatment (relative contribution in \%).

\begin{tabular}{|c|c|c|c|c|c|}
\hline & d 14 & d 23 & & d 35 & \\
\hline & & Low Zn & High Zn & Low Zn & High Zn \\
\hline k_Bacteria;p_Firmicutes;c_Bacilli;f_Lactobacillaceae;g_Lactobacillus & 75.0 & 63.7 & 73.1 & 74.9 & 50.0 \\
\hline k_Bacteria;p_Firmicutes;c_Bacilli;f_Streptococcaceae;g_Streptococcus & 5.8 & 11.3 & 9.3 & 11.4 & 10.1 \\
\hline k_Bacteria;p_Firmicutes;c_Bacilli;f_Turicibacteraceae;g__Turicibacter & 0.7 & 0.7 & 3.7 & 1.1 & 25.6 \\
\hline k_Bacteria;p_Actinobacteria;c_Actinobacteria;f_Actinomycetaceae;g_Actinomyces & 0.9 & 1.9 & 1.7 & 0.5 & 1.2 \\
\hline k_Bacteria;p_Firmicutes;c_Bacilli;Other;Other & 0.3 & 0.3 & 0.5 & 0.5 & 0.6 \\
\hline k_Bacteria;p_Firmicutes;c_Bacilli;f_Gemellaceae;g_Gemella & 0.4 & 0.3 & 0.3 & 0.6 & 0.8 \\
\hline k_Bacteria;p_Firmicutes;c_Bacilli;f_Streptococcaceae;g_Lactococcus & 0.0 & 0.1 & 0.0 & 0.1 & 0.2 \\
\hline k_Bacteria;p_Cyanobacteria;c_Chloroplast;f_g__ & 0.1 & 0.2 & 0.0 & 0.0 & 0.2 \\
\hline k_Bacteria;p_Firmicutes;c_Clostridia;f_Clostridiaceae;g_02d06 & 0.1 & 0.2 & 0.4 & 0.2 & 0.9 \\
\hline k_Bacteria;p_Firmicutes;c_Bacilli;f_Staphylococcaceae;g_Staphylococcus & 0.1 & 0.4 & 0.8 & 1.4 & 0.5 \\
\hline k_Bacteria;p_Firmicutes;c_Erysipelotrichi;f_Erysipelotrichaceae;g_[Eubacterium] & 0.7 & 0.6 & 1.3 & 0.5 & 0.6 \\
\hline k_Bacteria;p_Actinobacteria;c_Actinobacteria;Other;Other & 0.2 & 0.6 & 0.4 & 0.4 & 0.5 \\
\hline k_Bacteria;p_Firmicutes;c_Bacilli;f_Planococcaceae;g_Staphylococcus & 0.0 & 2.5 & 0.8 & 1.1 & 0.7 \\
\hline k_Bacteria;p_Firmicutes;c_Clostridia;f_Clostridiaceae;g_SMB53 & 0.1 & 0.2 & 0.2 & 0.1 & 0.8 \\
\hline k_Bacteria;p_Firmicutes;c_Erysipelotrichi;f_Erysipelotrichaceae;g__Bulleidia & 0.3 & 0.2 & 0.3 & 0.2 & 0.3 \\
\hline k_Bacteria;p_Firmicutes;c_Bacilli;f_Streptococcaceae;Other & 0.1 & 0.6 & 0.3 & 0.4 & 0.6 \\
\hline k_Bacteria;p_Firmicutes;c_Clostridia;f_Veillonellaceae;g_Veillonella & 3.7 & 5.9 & 1.7 & 0.2 & 0.1 \\
\hline k_Bacteria;p_Firmicutes;c_Clostridia;f_Veillonellaceae;g_Megasphaera & 0.8 & 0.3 & 0.1 & 0.1 & 0.3 \\
\hline k_Bacteria;p_Firmicutes;c_Bacilli;f_Leuconostocaceae;g_Weissella & 0.0 & 0.5 & 0.7 & 3.0 & 0.2 \\
\hline k_Bacteria;p_Firmicutes;c_Erysipelotrichi;f_Erysipelotrichaceae;Other & 0.1 & 0.3 & 0.2 & 0.1 & 0.1 \\
\hline k_Bacteria;p_Firmicutes;c_Clostridia;Other;Other & 0.1 & 0.5 & 0.1 & 0.0 & 0.2 \\
\hline k_Bacteria;p_Bacteroidetes;c_Bacteroidia;f_Prevotellaceae;g_Prevotella & 0.4 & 0.2 & 0.2 & 0.0 & 0.7 \\
\hline k_Bacteria;p_Actinobacteria;c_Actinobacteria;f_Corynebacteriaceae;g_Corynebacterium & 0.1 & 0.1 & 0.3 & 0.5 & 0.2 \\
\hline k_Bacteria;p_Firmicutes;c_Clostridia;f_Lachnospiraceae;g_Blautia & 0.2 & 0.2 & 0.1 & 0.1 & 0.1 \\
\hline k_Bacteria;p_Firmicutes;c_Clostridia;f_Lachnospiraceae;Other & 0.2 & 0.7 & 0.1 & 0.1 & 0.2 \\
\hline k_Bacteria;p_Firmicutes;c_Clostridia;f_Ruminococcaceae;Other & 0.1 & 0.4 & 0.1 & 0.0 & 0.1 \\
\hline k_Bacteria;p_Firmicutes;c_Clostridia;f_Veillonellaceae;g_Phascolarctobacterium & 0.1 & 0.4 & 0.0 & 0.0 & 0.1 \\
\hline k_Bacteria;p_Proteobacteria;c_Gammaproteobacteria;f_Pasteurellaceae;g_Actinobacillus & 0.2 & 0.5 & 0.0 & 0.0 & 0.0 \\
\hline k_Bacteria;p_Proteobacteria;c_Gammaproteobacteria;f_Pasteurellaceae;Other & 0.5 & 2.0 & 0.0 & 0.0 & 0.1 \\
\hline k_Bacteria;p_Proteobacteria;c_Gammaproteobacteria;f_Enterobacteriaceae;g_Escherichia & 2.3 & 0.1 & 0.0 & 0.0 & 0.1 \\
\hline k_Bacteria;p_Firmicutes;c_Bacilli;f_Staphylococcaceae;g_Jeotgalicoccus & 0.0 & 0.0 & 0.1 & 0.3 & 0.0 \\
\hline k_Bacteria;p_Proteobacteria;c_Gammaproteobacteria;f_Moraxellaceae;g__Acinetobacter & 0.4 & 0.0 & 0.0 & 0.0 & 0.0 \\
\hline k_Bacteria;p_Proteobacteria;c_Gammaproteobacteria;f_Moraxellaceae;g_Moraxella & 0.3 & 0.0 & 0.0 & 0.0 & 0.0 \\
\hline k_Bacteria;p_Proteobacteria;c_Gammaproteobacteria;f_Pasteurellaceae;g__Haemophilus & 0.4 & 0.1 & 0.0 & 0.0 & 0.0 \\
\hline k_Bacteria;p_Proteobacteria;c_Betaproteobacteria;f_Neisseriaceae;Other & 0.8 & 0.0 & 0.0 & 0.0 & 0.0 \\
\hline k_Bacteria;P_Proteobacteria;c_Gammaproteobacteria;f_Moraxellaceae;Other & 0.3 & 0.0 & 0.0 & 0.0 & 0.0 \\
\hline k_Bacteria;p_Proteobacteria;c_Epsilonproteobacteria;f_Helicobacteraceae;g_Flexispira & 0.0 & 0.2 & 0.0 & 0.0 & 0.0 \\
\hline k_Bacteria;P_Bacteroidetes;c_Bacteroidia;f_Porphyromonadaceae;Other & 0.5 & 0.0 & 0.0 & 0.0 & 0.0 \\
\hline k_Bacteria;p_Bacteroidetes;C_Flavobacteriia;f_[Weeksellaceae];g__ & 0.7 & 0.0 & 0.0 & 0.0 & 0.0 \\
\hline k_Bacteria;p_Firmicutes;c_Clostridia;f_[Tissierellaceae];g_Anaerococcus & 0.0 & 0.0 & 0.0 & 0.0 & 0.2 \\
\hline
\end{tabular}


Appendix 2. Overview of microbiota composition at genus level in ileal digesta as affected by age (time) and dietary treatment (relative contribution in \%).

\begin{tabular}{|c|c|c|c|c|c|}
\hline & d 14 & d 23 & & d 35 & \\
\hline & & Low Zn & High Zn & Low Zn & High Zn \\
\hline k_Bacteria;p_Firmicutes;c_Bacilli;f_Lactobacillaceae;g_Lactobacillus & 77.0 & 77.2 & 53.5 & 37.9 & 30.6 \\
\hline k_Bacteria;p_Firmicutes;c_Bacilli;f_Streptococcaceae;g_Streptococcus & 4.5 & 10.1 & 15.2 & 5.2 & 10.9 \\
\hline k_Bacteria;p_Firmicutes;c_Bacilli;f_Planococcaceae;g__Staphylococcus & 0.2 & 0.3 & 1.8 & 1.0 & 0.5 \\
\hline k_Bacteria;p_Actinobacteria;c_Actinobacteria;f_Actinomycetaceae;g_Actinomyces & 1.3 & 0.7 & 1.9 & 0.3 & 0.6 \\
\hline k_Bacteria;p_Firmicutes;c_Bacilli;f_Staphylococcaceae;g_Staphylococcus & 0.1 & 0.2 & 1.2 & 0.7 & 0.2 \\
\hline k_Bacteria;p_Firmicutes;c_Clostridia;f_Veillonellaceae;g_Veillonella & 2.6 & 4.5 & 1.2 & 0.4 & 0.5 \\
\hline k_Bacteria;p_Firmicutes;c_Bacilli;Other;Other & 0.2 & 0.3 & 0.6 & 0.2 & 0.3 \\
\hline k_Bacteria;p_Firmicutes;c_Bacilli;f_Turicibacteraceae;g__Turicibacter & 0.7 & 2.0 & 10.4 & 30.9 & 34.6 \\
\hline k_Bacteria;p_Firmicutes;c_Bacilli;f_Streptococcaceae;Other & 0.1 & 0.3 & 0.8 & 0.5 & 0.5 \\
\hline k_Bacteria;p_Proteobacteria;c_Epsilonproteobacteria;f_Helicobacteraceae;g_Flexispira & 0.0 & 0.0 & 0.0 & 0.3 & 0.1 \\
\hline k_Bacteria;p_Firmicutes;c_Bacilli;f_Enterococcaceae;Other & 2.6 & 0.1 & 0.3 & 0.2 & 0.0 \\
\hline k_Bacteria;p_Proteobacteria;c_Gammaproteobacteria;f_Pasteurellaceae;Other & 0.4 & 0.1 & 4.9 & 8.1 & 11.0 \\
\hline k_Bacteria;p_Firmicutes;c_Bacilli;f_Leuconostocaceae;g_Weissella & 0.0 & 0.3 & 1.1 & 3.0 & 0.7 \\
\hline k_Bacteria;p_Chlamydiae;c_Chlamydiia;f_Chlamydiaceae;Other & 0.0 & 0.1 & 0.3 & 0.5 & 0.1 \\
\hline k_Bacteria;p_Proteobacteria;c_Gammaproteobacteria;f_Pasteurellaceae;g__Actinobacillus & 0.1 & 0.1 & 2.1 & 1.8 & 2.8 \\
\hline k_Bacteria;p_Firmicutes;c_Clostridia;f_Clostridiaceae;g_02d06 & 0.1 & 0.0 & 0.4 & 1.3 & 0.9 \\
\hline k_Bacteria;p_Proteobacteria;c_Gammaproteobacteria;f_Enterobacteriaceae;g_Escherichia & 6.5 & 1.5 & 0.4 & 1.3 & 1.1 \\
\hline k_Bacteria;p_Firmicutes;c_Clostridia;f_Clostridiaceae;g_SMB53 & 0.1 & 0.0 & 0.1 & 0.3 & 0.9 \\
\hline k_Bacteria;p_Proteobacteria;c_Gammaproteobacteria;f_Pasteurellaceae;g__Haemophilus & 0.0 & 0.0 & 0.4 & 0.7 & 1.2 \\
\hline k_Bacteria;p_Bacteroidetes;c_Bacteroidia;f__Prevotellaceae;g_Prevotella & 0.0 & 0.2 & 0.1 & 0.5 & 0.1 \\
\hline k_Bacteria;p_Firmicutes;c_Clostridia;f_Clostridiaceae;g_Clostridium & 0.0 & 0.0 & 0.0 & 0.7 & 0.3 \\
\hline k_Bacteria;p_Proteobacteria;c_Gammaproteobacteria;f_Enterobacteriaceae;g__Providencia & 0.4 & 0.0 & 0.0 & 0.2 & 0.0 \\
\hline k_Bacteria;p_Proteobacteria;c_Gammaproteobacteria;f_Pasteurellaceae;g_Aggregatibacter & 0.0 & 0.0 & 0.0 & 0.3 & 0.4 \\
\hline
\end{tabular}


Appendix 3. Overview of taxa at family level showing a difference in relative contribution at $\mathrm{P}<0.05$ between dietary $\mathrm{Zn}$ treatment (group) $\mathrm{x}$ day (time/age), with the constraint of their overall contribution being at least $1 \%$ of the total microbiota over digesta samples of jejunal or ileal origin.
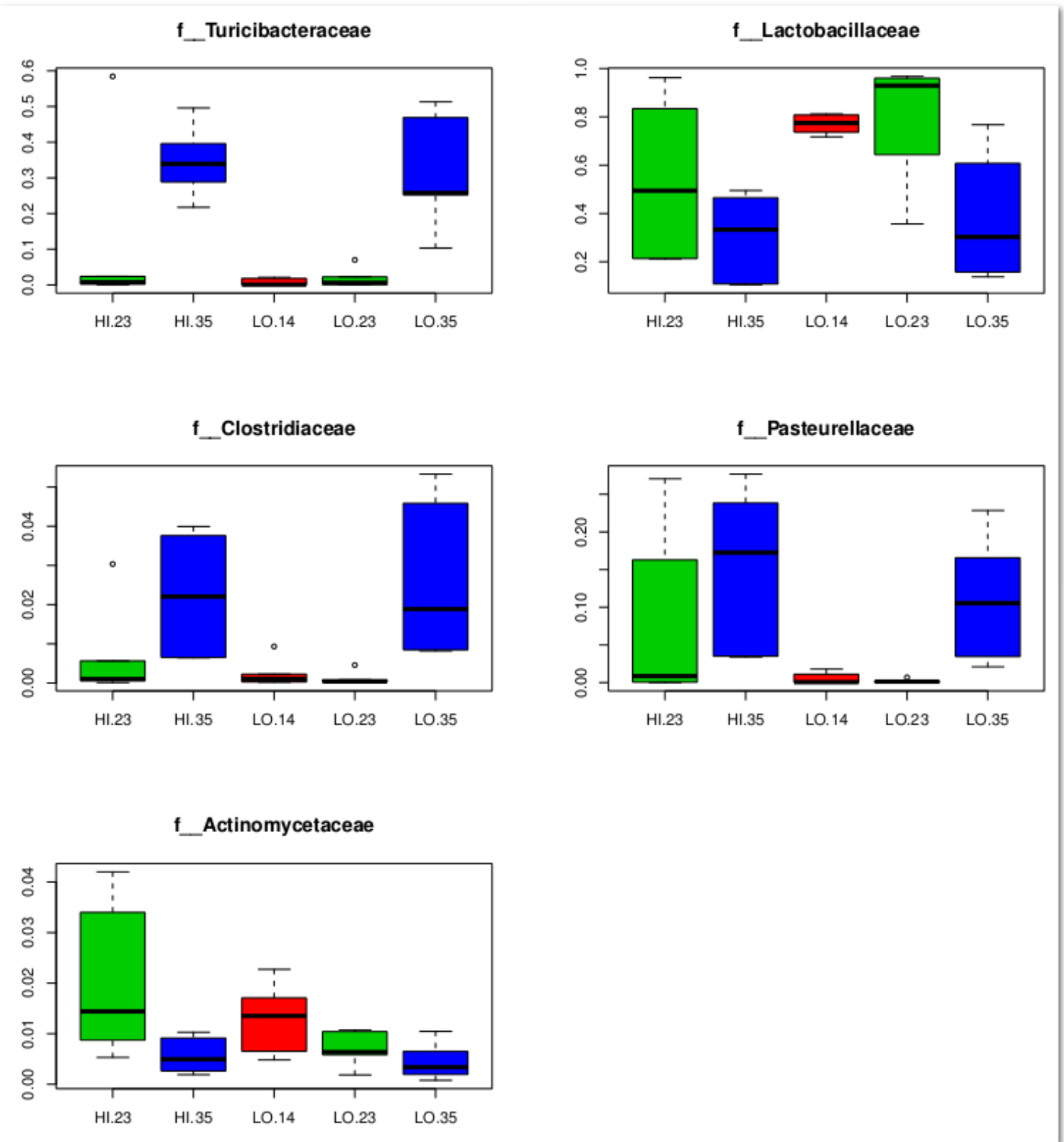
Appendix 4. Overview of taxa at genus level showing a difference in relative contribution at $\mathrm{P}<0.05$ between dietary $\mathrm{Zn}$ treatment (group) $\mathrm{x}$ day (time/age), with the constraint of their overall contribution being at least $1 \%$ of the total microbiota over digesta samples of jejunal or ileal origin.
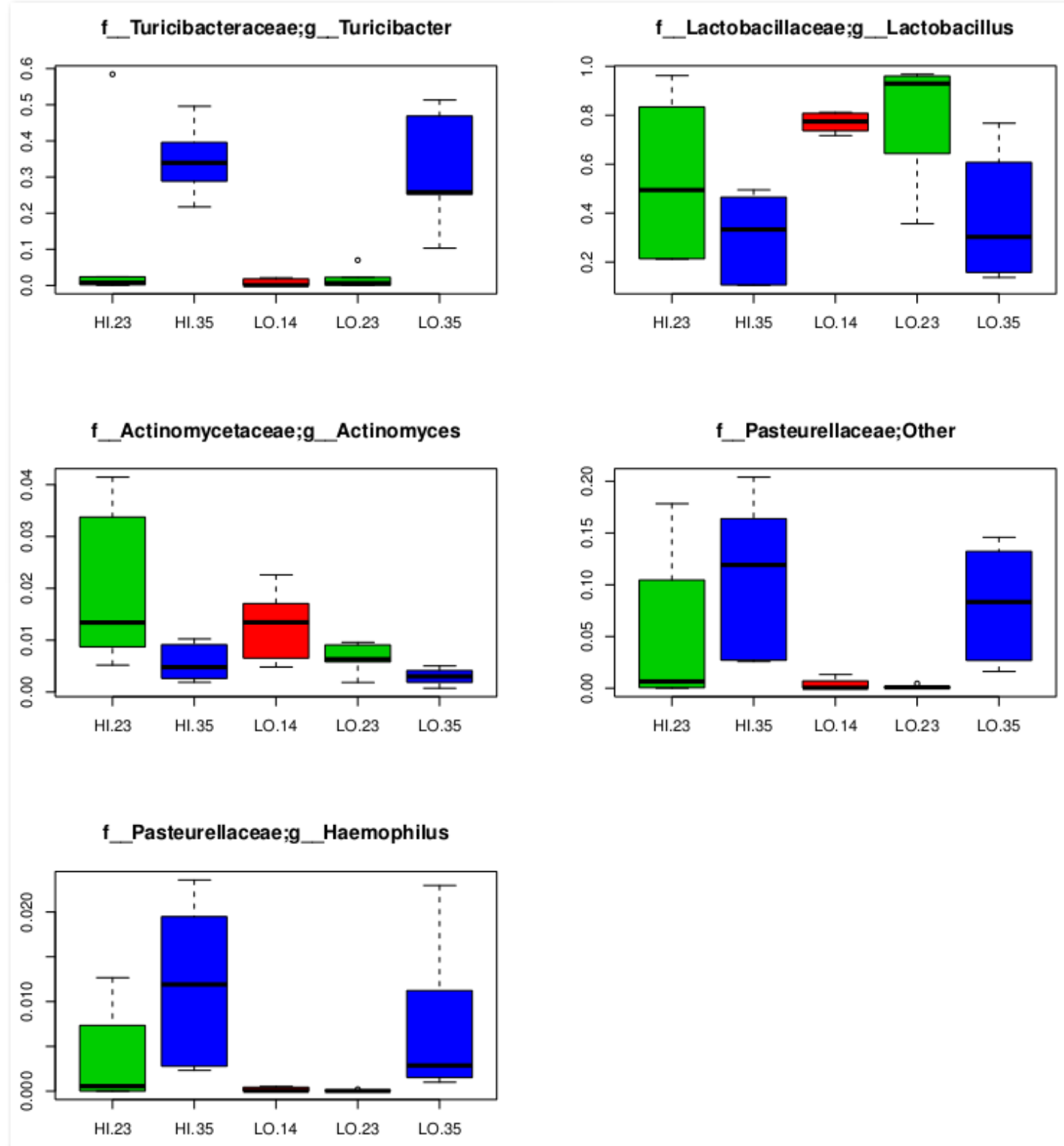
Appendix 5. Overview of taxa at species level showing a difference in relative contribution at $\mathrm{P}<0.05$ between dietary $\mathrm{Zn}$ treatment (group) $\mathrm{x}$ day (time/age), with the constraint of their overall contribution being at least $1 \%$ of the total microbiota over digesta samples of jejunal or ileal origin.

f__Turicibacteraceae;g__Turicibacter;s__

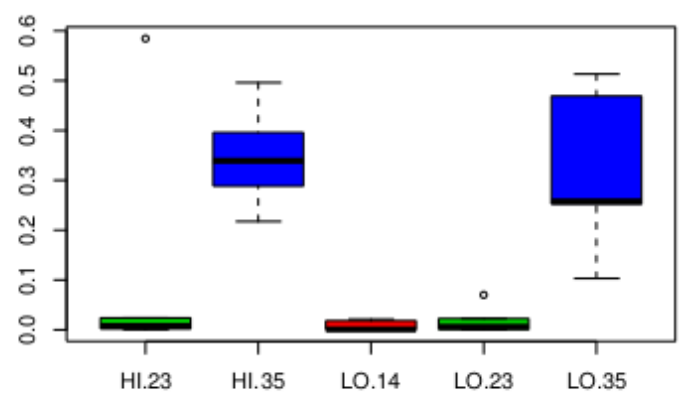

f__Actinomycetaceae;g__Actinomyces;s_

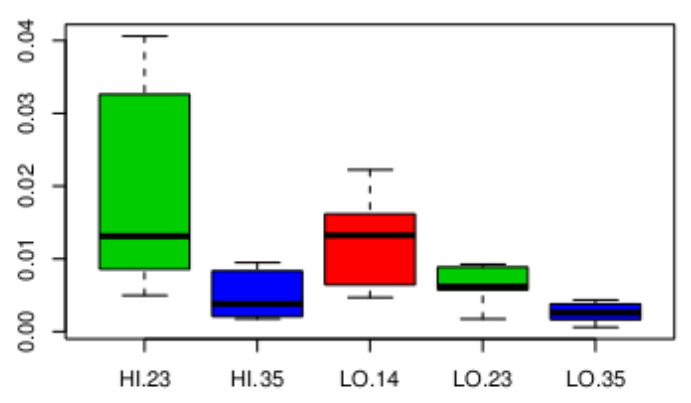

f__Lactobacillaceae;g__Lactobacillus;s_agilis

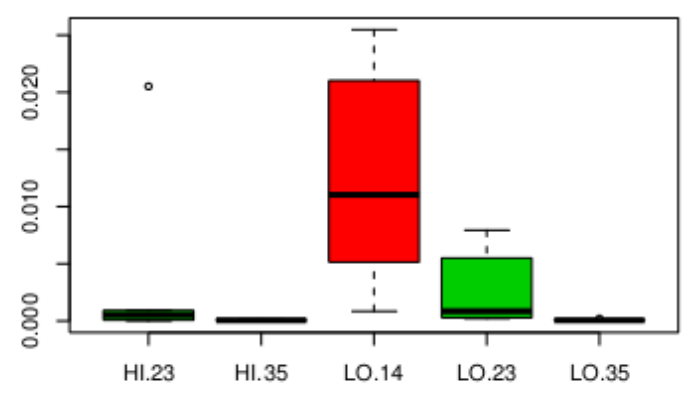

f_Lactobacillaceae;g__Lactobacillus;s__

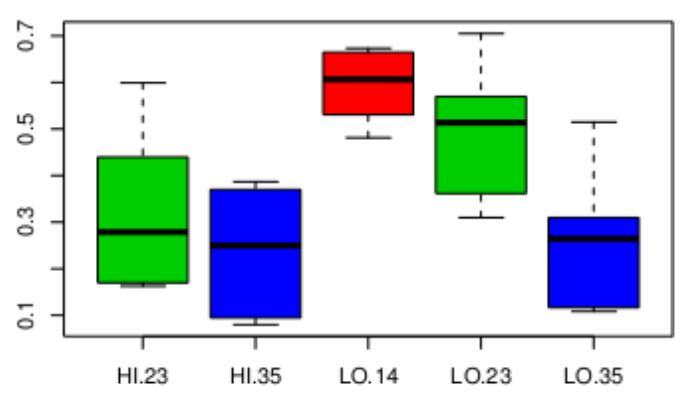

f__Pasteurellaceae;Other;Other

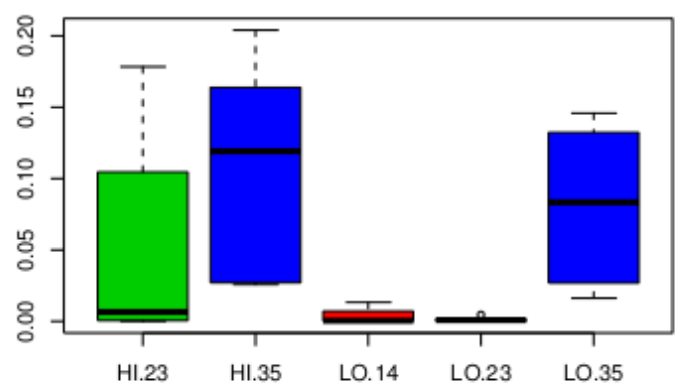

f__Pasteurellaceae;g__Haemophilus;s__parasuis

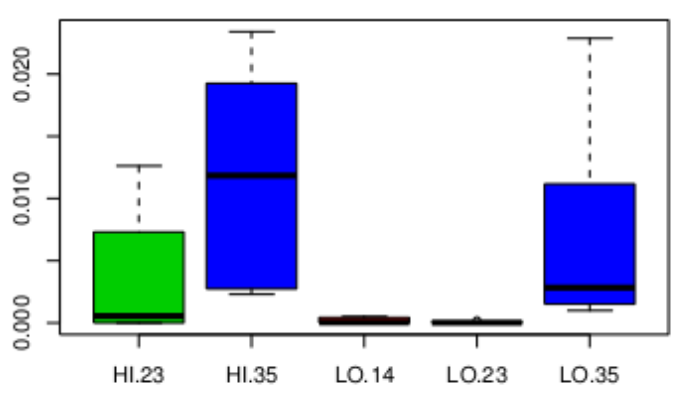



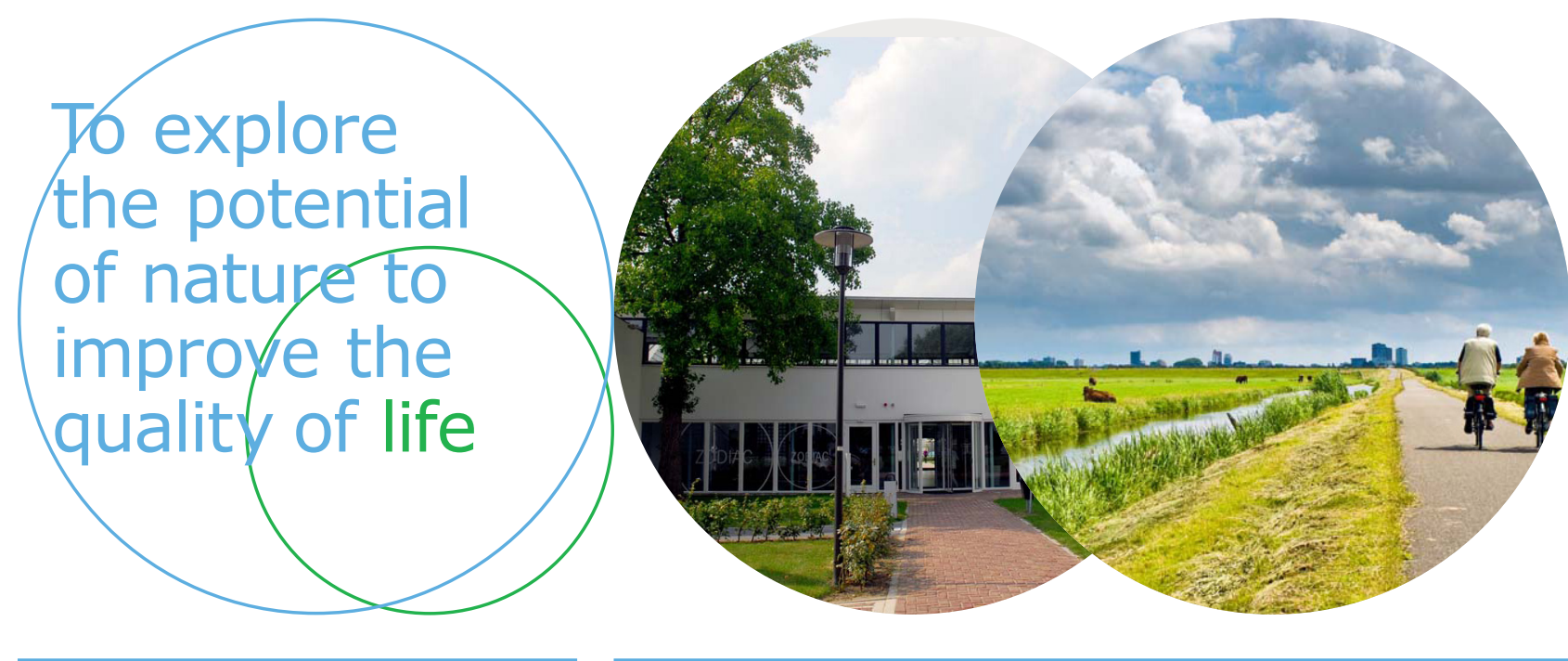

Wageningen Livestock Research P.O. Box 338

$6700 \mathrm{AH}$ Wageningen

The Netherlands

$\mathrm{T}+31(0) 317483953$

E info.livestockresearch@wur.nl www.wur.nl/livestock-research
Together with our clients, we integrate scientific know-how and practical experience to develop livestock concepts for the 21st century. With our expertise on innovative livestock systems, nutrition, welfare, genetics and environmental impact of livestock farming and our state-of-the art research facilities, such as Dairy Campus and Swine Innovation Centre Sterksel, we support our customers to find solutions for current and future challenges.

The mission of Wageningen University \& Research is 'To explore the potential of nature to improve the quality of life'. Within Wageningen University, nine specialised research institutes of the DLO Foundation have joined forces with Wageningen University to help answer the most important questions in the domain of healthy food and living environment. With approximately 30 locations, 6,000 members of staff and 10,000 students, Wageningen University is one of the leading organisations in its domain worldwide. The integral approach to problems and the cooperation between the various disciplines are at the heart of the unique Wageningen Approach. 